\title{
Two-Carbon Ring Enlargement of Five-, Six-, and Seven-Membered 1-Aza-2-vinylcycloalk-2-enes with Dimethyl Acetylenedicarboxylate and Subsequent Thermal Isomerization Reactions*
}

\author{
Gerhard Maas, Robert Reinhard, and Hans-Georg Herz \\ Abteilung Organische Chemie I, Universität Ulm, Albert-Einstein-Allee 11, D-89081 Ulm \\ Reprint requests to Prof. Dr. G. Maas. Fax: +49(731)5022803. E-mail: gerhard.maas@uni-ulm.de
}

Z. Naturforsch. 61b, 385 - 395 (2006); received October 6, 2005

2-Aminodienes, in which the enamine function is incorporated in a five-, six-, or seven-membered ring, react with dimethyl acetylenedicarboxylate in a sequence of [2+2] cycloaddition and electrocyclic ring-opening to form the two-carbon ring expanded unsaturated heterocycles, i.e., 3,4dicarboxylate substituted 6,7-dihydro- $1 H$-azepines $\mathbf{3}, \mathbf{8}$ and 21, 1,6,7,8-tetrahydroazocines 22, and 6,7,8,9-tetrahydro- $1 H$-azonines 13. Similarly, 2-[(2-thienyl)ethynyl]-4,5,6,7-tetrahydro- $1 H$-azepine $\mathbf{9}$ is converted into 2-[(2-thienyl)ethynyl]-6,7,8,9-1H-azonine-3,4-dicarboxylate $\mathbf{1 0}$ which was characterized by X-ray structure determination. The eight- and nine-membered azaheterocycles $\mathbf{2 2}$ and 13, which have not been isolated, undergo thermal isomerization at elevated temperatures. Thus, ring contraction by a $6 \pi$-electrocyclic reaction takes place for $N$-methyl substituted azonine 13, while the $N$-allyl moiety of azocines 22 engages in an intramolecular Diels-Alder reaction or a 1,7electrocyclization reaction.

Key words: Enamines, 2-Aminodienes, Medium-Sized Aza Heterocycles, Ring Enlargement, Ring Contraction

\section{Introduction}

The two-carbon ring expansion of enamines derived from cyclic ketones (i.e. 1-(dialkylamino)cycloalkenes) with electron-deficient alkynes, in particular acetylenic esters, has often been applied to the synthesis of medium-sized carbocyclic and heterocyclic compounds (Scheme 1, type I); see lit. [1] and cited references. Careful investigations have shown that in an unpolar solvent, [2+2] cycloaddition initially generates 3-amino-cyclobutenes which undergo conrotatory ring opening to form cis,trans-cycloalkadienes [1]. The condensed cyclobutenes derived from five- and six-membered enamines could be isolated under careful work-up conditions and were found to rearrange slowly into the cis,cis-cycloalkadienes, while the latter were obtained directly from larger-sized aminocycloalkenes. The cis,trans-dienes underwent thermal isomerization to form cis, cis-cycloalkadienes more or less readily, depending on the ring size.

\footnotetext{
* Presented in part at the $7^{\text {th }}$ Conference on Iminium Salts (ImSaT-7), Bartholomä/Ostalbkreis, September 6-8, 2005.
}

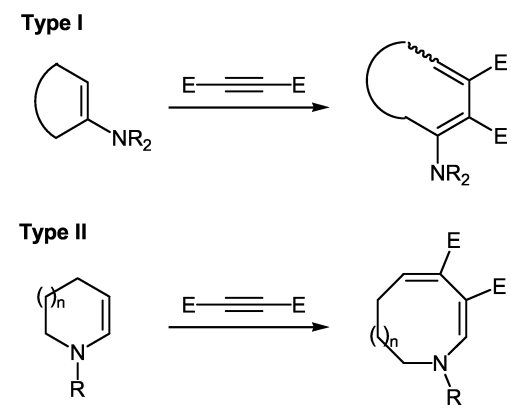

Scheme 1. Ring expansion of 1-amino-cycloalkenes (type 1) and 1-aza-2-cycloalkenes (type 2 ) with acetylenedicarboxylates $\left(\mathrm{E}=\mathrm{CO}_{2} \mathrm{Me}\right)$.

The two-carbon ring expansion strategy can also be used to convert endocyclic enamines into unsaturated seven-, eight-, and nine-membered azaheterocycles (Scheme 1, type II) [2-5]. Some years ago, we have worked out a method to prepare 1-aza-2-vinylcycloalk-2-enes, i.e., semicyclic 2-amino-1,3-dienes, with the enamine functionality incorporated in five-, six-, and seven-membered rings [6]. We report now on the ring expansion of these cyclic enamines with dimethyl acetylenedicarboxylate and on subsequent thermally induced isomerization reactions [7]. 


\section{Results and Discussion}

The 2-alkenyl-4,5-dihydropyrroles $\mathbf{1 a - c}$ were prepared from the corresponding 1-((het)aryl)-2-(1-methylpyrrolidin-2-ylidene)ethan-1-one in three steps [6]. The reaction with dimethyl acetylenedicarboxylate (DMAD) was conducted in diethyl ether below room temperature to obtain the bicyclic [2+2] cycloaddition products 2 (Scheme 2). However, the latter could not be separated from the reaction mixture. When the work-up was done by Kugelrohr distillation, yellow oils distilled at $\geq 200{ }^{\circ} \mathrm{C} / 0.005$ mbar which yielded yellow crystals $(45-65 \%$ yield) that were identified as 6,7-dihydro- $1 H$-azepine-3,4-dicarboxylates 3a-c. On the other hand, work-up of the reaction mixture obtained from 1a, $\mathbf{b}$ by column chromatography over silica gel yielded the (dihydropyrrolyl)maleates or fumarates $\mathbf{4 a}, \mathbf{b}$; since only one diastereomer was obtained, the available NMR data did not allow to assign the configuration at the ester-substituted olefinic bond $(\delta(=\mathrm{CH})=4.92 \mathrm{ppm})$. Thus, the initially formed cycloaddition products 2 undergo the expected electrocyclic cyclobutene ring-opening/ring expansion reaction on thermal impact, while the formation of dihydropyrroles $\mathbf{4}$ can be interpreted as a proton-catalyzed ring-opening of the push-pull-substituted cyclobutene

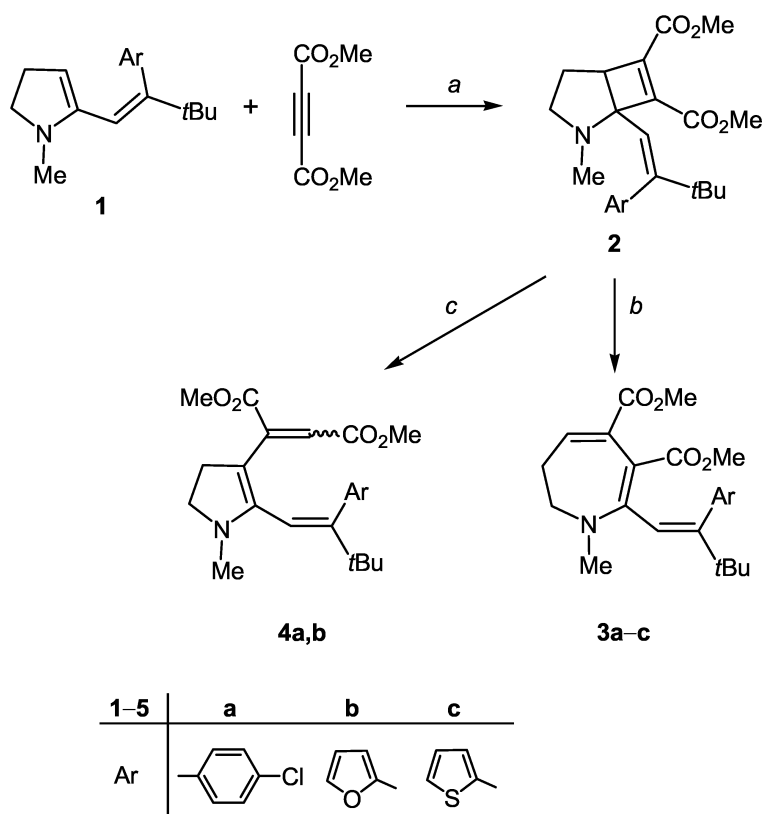

Scheme 2. Reaction conditions: a) $\mathrm{Et}_{2} \mathrm{O},-40{ }^{\circ} \mathrm{C} \rightarrow$ r.t.; b) no solvent, bulb-to-bulb distillation at $200-240{ }^{\circ} \mathrm{C} /$ 0.005 mbar $(45-62 \%$ yield from $\mathbf{1 a - c}) ; c)$ chromatographic work-up (silica gel) $(76-82 \%$ yield from 1a, b).

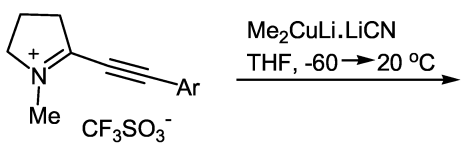

5

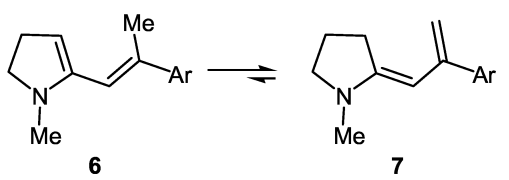

1) $\mathrm{MeO}_{2} \mathrm{C}=\mathrm{CO}_{2} \mathrm{Me}$

$$
\text { ether, }-40^{\circ} \mathrm{C} \text { to r.t. }
$$

2) no solvent, $200^{\circ} \mathrm{C} / 0.005$ mbar<smiles>COC(=O)c1c(C=C(Br)Br)c(C(C)=O)c(C(C)=O)n1C</smiles><smiles>Cc1ccco1</smiles>

Scheme 3. Synthesis of aminodienes $6 / 7$ and reaction with DMAD. Yield of 8: $56 \%$ from 5 .

substructure. Analogous reactions take place when $[2+2]$ cycloaddition products of 1-dialkylamino-cycloalkenes and acetylenic esters are dissolved in methanol or $\mathrm{CDCl}_{3}$ [1a, 1b, 8, 9].

When the tert-butyl group in aminodienes $\mathbf{1}$ is replaced by a methyl group, a tautomeric equilibrium between the semicyclic 2-aminodiene form 6 and the exocyclic 1-aminodiene form 7 is possible (Scheme 3). In contrast to the tert-butyl analogues 1, 1-aminodiene 7 (Ar = 4-chlorophenyl and 2-thienyl) has been observed exclusively by ${ }^{1} \mathrm{H}$ NMR spectroscopy [6]. The same is true for the 2-furyl substituted aminodiene (7, $\mathrm{Ar}=2$ furyl) which we have now prepared by dimethylcuprate addition to propyne iminium salt 5 (Scheme 3 ). Since 7 could not be isolated in pure form, the crude product was directly combined with DMAD and the mixture was subsequently heated at $200{ }^{\circ} \mathrm{C}$. By analogy to the transformation $\mathbf{1} \rightarrow \mathbf{3}$, dihydroazepine $\mathbf{8}$ was isolated in $58 \%$ yield. This result suggests indeed that DMAD does not react with the 1 -aminodiene 7 but rather with the minute amount of the semicyclic aminodiene 6 which is in dynamic equilibrium with 7.

A $7 \rightarrow 9$ ring expansion took place when the 2-[(2thienyl)ethynyl]-4,5,6,7-tetrahydro- $1 H$-azepine 9 [10] was exposed to DMAD at $\leq 20{ }^{\circ} \mathrm{C}$ (Scheme 4). Extraction of the reaction mixture with ether and crystallization yielded the tetrahydro- $1 H$-azoninedicarboxylate $\mathbf{1 0}$ in $83 \%$ yield. Again, the initial [2+2] cycloaddition product was not observed because the electro- 


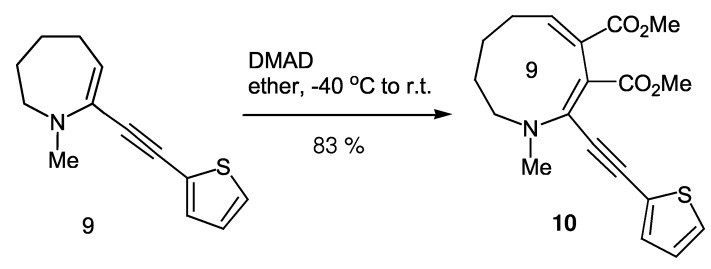

Scheme $4.7 \rightarrow 9$ Ring expansion of tetrahydroazepine $\mathbf{9}$.

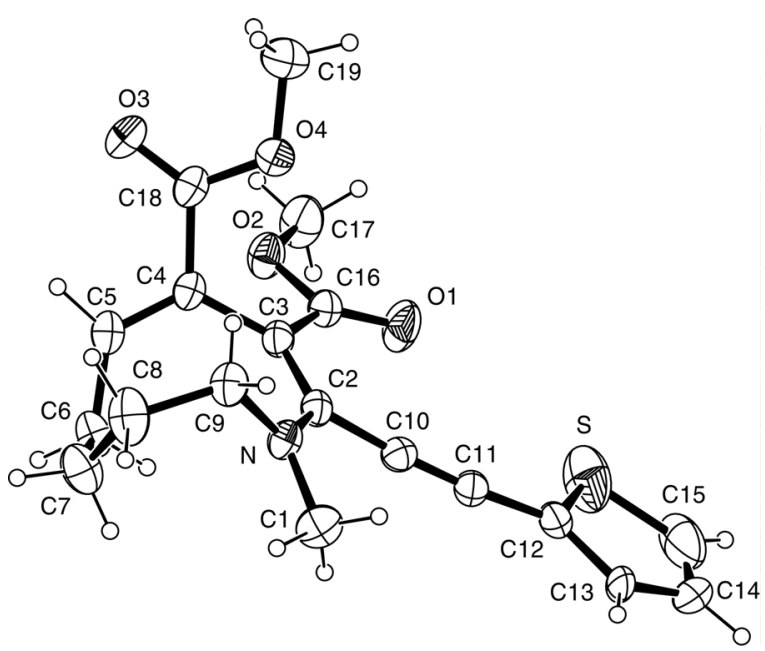

Fig. 1. Solid-state structure of 10; ellipsoids of thermal vibration are shown at the $30 \%$ probability level. Selected bond lengths $[\AA]$ and torsion angles $\left[{ }^{\circ}\right]$ : N-C2 1.367(6), C2-C3 1.375(7), C3-C16 1.470(7), C3-C4 1.486(6), C4-C18 1.504(7), C4-C5 1.334(7), C2-C10 1.441(7), C10-C11 1.195(7); C9-N-C2-C3 - 25.4(7), N-C2-C3-C4 -5.6(8), C2-C3-C4-C5 $-54.3(7)$. The thiophene ring is probably disordered over two position, with a minor component related to the major one by a $180^{\circ}$ rotation around the $\mathrm{C} 11-\mathrm{C} 12$ bond. However, this disorder model was not included in the refinement.

cyclic ring-opening took place already at room temperature or below. The NMR data of $\mathbf{1 0}$ did not allow to firmly establish the configuration of the double bonds in the azonine ring. A crystal structure determination revealed the cis,cis-configuration of $\mathbf{1 0}$ (Fig. 1). It is interesting to note that the reaction of 1-dialkylaminocycloheptenes and -cyclooctenes with DMAD at room temperature generates the expected two-carbon ringexpanded cycloalka-1,3-dienes with cis,trans configuration [1] which have been isolated and were transformed into the cis,cis-isomer on heating. Thus, it is not clear whether cis,cis-10 is formed via the cis,transisomer, the latter resulting from an orbital symmetryallowed conrotatory ring-opening of the initial [2+2] cycloaddition product (see Introduction). The possibility has been discussed that push-pull substituted

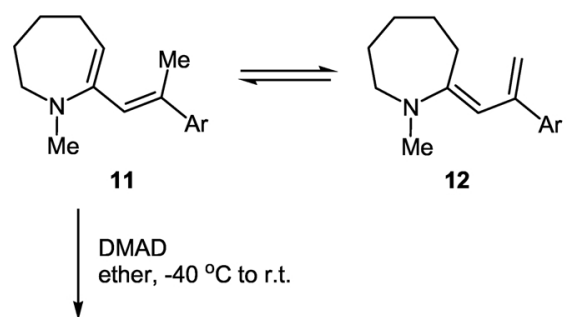

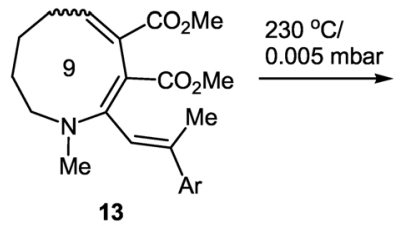

$A r=4$

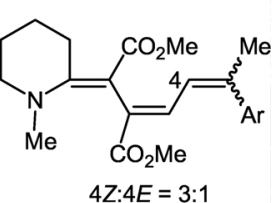

14

Scheme $5.7 \rightarrow 9$ Ring expansion of dihydroazepine $\mathbf{1 1}$ and subsequent thermal isomerization. Yield of 14: $61 \%$ from 11/12.

3-aminocyclobutene-1-carboxylates may undergo the ring opening by a disrotatory concerted electrocyclic process [11] or stepwise via a dipolar intermediate [12] (see also lit. [1]).

The crystal structure analysis of $\mathbf{1 0}$ shows that the tetrahydro- $1 H$-azonine ring adopts a distorted boat conformation (Fig. 1). The dienamine moiety is not fully conjugated, because the torsion angle between the two double bonds (C2-C3-C4-C5) arises to $-54.3^{\circ}$. On the other hand, the torsion angles of the enaminoester moiety $\mathrm{N}-\mathrm{C}=\mathrm{C}-\mathrm{COOMe}$ allow extended $\pi$ conjugation with the expected bond length changes due to the push-pull character of this conjugated system.

In contrast to the five-membered enamine $\mathbf{6}$, the seven-membered analogue $\mathbf{1 1}$ is the major component in the tautomeric equilibrium with the 1-aminodiene form 12 (11:12 $\approx 2.7: 1)$ [6] (Scheme 5). Trapping of the more reactive 2-aminodiene form 11 with DMAD was expected to furnish tetrahydroazonine $\mathbf{1 3}$ by analogy with the conversion $\mathbf{9} \rightarrow \mathbf{1 0}$. As $\mathbf{1 3}$ could not be isolated in pure form from the reaction mixture by crystallization or chromatography, a bulb-to-bulb distillation was applied which unexpectedly gave the acyclic hexatriene $\mathbf{1 4}$ as a 3:1 mixture of diastereomers. The structure of $\mathbf{1 4}$ was elucidated by X-ray diffraction analysis of a crystal taken from the mixture of diastereoisomers (Fig. 2). The ${ }^{1} \mathrm{H}$ chemical shifts of the two diastereomers are quite similar except for the significantly different values of the olefinic proton at the thienyl-substituted double bond. The chemical shift 


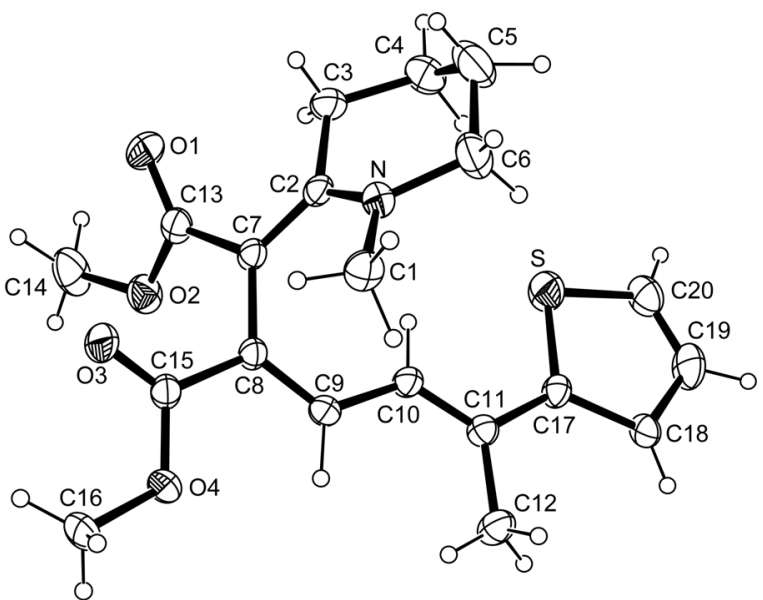

Fig. 2. Solid-state structure of $(1 E, 2 E, 4 E)-\mathbf{1 4}$; ellipsoids of thermal vibration are shown at the $30 \%$ probability level. Selected bond lengths $[\AA]$ and torsion angles $\left[{ }^{\circ}\right]$ : $\mathrm{N}-\mathrm{C} 2$ 1.352(4), C2-C7 1.379(5), C7-C13 1.466(5), C7-C8 $1.480(4)$, C $8-\mathrm{C} 15$ 1.492(4), C8-C9 1.355(5), C9-C10 1.435(4), C10-C11 1.343(5); N-C2-C7-C8 -22.1(5), C2C7-C8-C9 -52.2(5), C7-C8-C9-C10 -0.9(5), C8-C9C10-C11 172.1(4), C9-C10-C11-C17 179.3(3), C10-C11C17-C18 172.3(3). The thiophene ring is probably disordered over two position, with a minor component related to the major one by a $180^{\circ}$ rotation around the exocyclic $\mathrm{C}-\mathrm{C}$ bond. However, this disorder model was not included in the refinement.

of this proton is found at $\delta=6.27 \mathrm{ppm}$ for the major diastereomer and at $\delta=6.62 \mathrm{ppm}$ for the minor, corresponding to the $Z$ and $E$ configuration, respectively, in line with the assignment made for the starting material, aminodiene 11 [6]. Thus, a partial geometrical isomerization of the thienyl-substituted double bond has occurred, and the crystal structure has been determined for the minor diastereoisomer $1 E, 2 E, 4 E-14$.

The formation of piperidinylidene-hexatriene $\mathbf{1 4}$ can be explained by a thermally induced isomerization of azonine $\mathbf{1 3}$ (Scheme 6). Ring contraction by transannular formation of an $\mathrm{N}-\mathrm{C}$ bond yields betaine 15A which rearranges to ylide $15 \mathrm{~B}$ by a hydrogen shift. Ring-opening of the cyclopentene ring finally generates 14. It should be noted that this rearrangement can occur by a series of three concerted pericyclic processes with defined stereochemistry: disrotatory $6 \pi$ electrocyclization of $\mathbf{1 3}$, suprafacial $[1,4-\mathrm{H}]$ shift of $\mathbf{1 5 a}$, and disrotatory six-electron ring opening of 15B. In order to arrive at the observed $E(2,3)$ configuration of triene $\mathbf{1 4}$, this sequence must begin with the cis,trans-azonine $\mathbf{1 3}$ which undergoes electrocyclization to form trans-fused 15A. However, we have no spectroscopic proof of the double bond con-
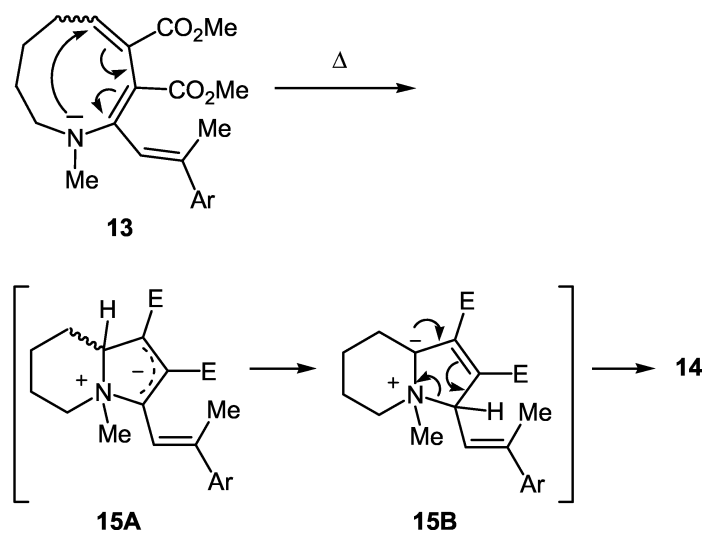

Scheme 6. Possible mechanism for the formation of triene 14; $\mathrm{Ar}=2$-thienyl, $\mathrm{E}=\mathrm{CO}_{2} \mathrm{Me}$.

figuration of $\mathbf{1 3}$, and the case of azonine 10, as described above, shows that the cis,cis-form is formed already at room temperature. The electrocyclic ring contraction of cis,cis-13a would generate cis-fused 15A; a suprafacial $\mathrm{H}$ shift of the latter would lead to transdisubstituted 15b from which $\mathrm{Z}(3,4)-\mathbf{1 4}$ would result after disrotatory opening of the $\mathrm{N}-\mathrm{C}$ bond in the fivemembered ring. Thus, if the thermal isomerization really starts with cis,cis-azonine 13, at least one of the three steps cannot be under the predicted stereochemical control. For example, an isomerization of cis-fused 15a to trans-disubstituted $\mathbf{1 5 b}$ could be caused by (intermolecular) proton transfer, and the ring opening of $\mathbf{1 5 b}$ could be a thermally induced homolytic process.

The ring contraction $\mathbf{1 3} \rightarrow \mathbf{1 5 a}$ could also be considered as a transannular nucleophilic addition of the ring nitrogen atom to the electron-deficient 4,5-double bond. The nucleophilicity of the nitrogen atom may be considered too low because of the partial delocalization of its lone pair of electrons in the enaminoester moiety (see the structure of $\mathbf{1 0}$ discussed above), but conformational changes at elevated temperatures may change this situation. Transannular interactions of medium-sized azaheterocycles with carbonyl functions are known [13] and have been studied by molecular mechanics calculations [14]. Transannular cyclization reactions such as the electrophile-induced cyclization of unsaturated nine- and ten-membered $N$-benzyl lactams [15] and $N$-nucleophilic epoxide-ring opening reactions of nine-membered ring lactams [16] have recently been used for the synthesis of quinolizidine and indolizidine ring systems.

Next, we were interested to learn whether the presence of an allyl instead of a methyl substituent on the 


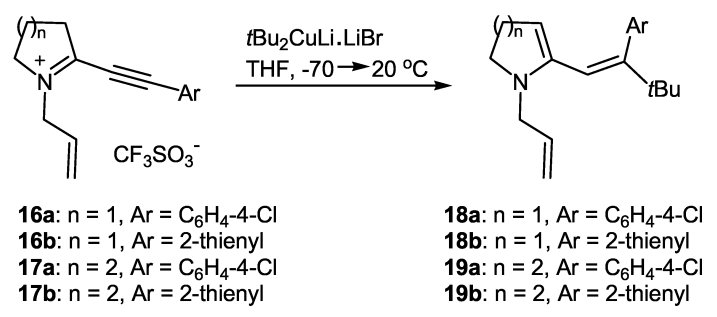

Scheme 7. Preparation of semicyclic N-allyl-aminodienes 18 and 19.

nitrogen atom of compounds such as dihydroazepines 3 would open other pathways for thermal isomerization reactions. To this end, we prepared $N$-allyl-2,3dihydropyrroles 18 and $N$-allyl-1,2,3,4-tetrahydropyridines 19 by organocuprate addition to the semicyclic propyniminium triflates 16 and 17 [17], respectively (Scheme 7). In all cases, only one diastereoisomer was found to which the $E$ configuration of the tert-butylsubstituted $\mathrm{C}=\mathrm{C}$ bond could be assigned by NOE NMR experiments.

As none of the 2-aminodienes 18 and $\mathbf{1 9}$ could be isolated in pure form, the crude products were treated directly with DMAD at $\leq 20{ }^{\circ} \mathrm{C}$. Starting from 18a, $\mathbf{b}$ and by full analogy with the behavior of the $N$-methyl analogues 1 (see Scheme 2), the expected initial cycloaddition products $\mathbf{2 0}(\mathrm{n}=1)$ were not isolated and the crude product mixture was heated in toluene at $120{ }^{\circ} \mathrm{C}$ (Scheme 8). This procedure yielded the 6,7-dihydro- $1 H$-azepine-3,4-dicarboxylates $21 \mathbf{a}, \dot{\mathbf{b}}$ in 47 and $52 \%$ yield. The characteristic NMR data match those of $3 \mathbf{a}-\mathbf{c}$. In addition, the allyl $\mathrm{NCH}_{2}$ protons were found to be diastereotopic which indicated the persistance of a chiral conformation. As the strongly broadened signals of both the o,o'- and m,m'-CH groups in 21a indicated that these nuclei were on the way to chemical nonequivalence, it can be concluded that steric hindrance between the $N$-allyl substituent and the alkenyl group attached to $\mathrm{C}-2$ restricts the free rotation around the ring/substituent bonds.

The reaction of aminodienes 19a, b with DMAD and subsequent heating at $160{ }^{\circ} \mathrm{C}$ in toluene did not furnish the expected tetrahydroazocines $\mathbf{2 2}$ (Scheme 8). From 4-chlorophenyl-substituted 19a, the azepino[1,2a] azocine $\mathbf{2 4}$ was obtained in $68 \%$ yield (relative to the precursor of 19a, iminium salt 17a), while thienylsubstituted 19b gave the tetracyclic azocino[2,1-a]thieno[3,2-f] isoindole $\mathbf{2 5}$ in $42 \%$ yield. The structure of 24 and 25 was established by X-ray crystal structure determination (Figs 3 and 4) and all ${ }^{1} \mathrm{H}$ and ${ }^{13} \mathrm{C}$ NMR chemical shifts were assigned from $1 \mathrm{D}$ and $2 \mathrm{D}$ spec-

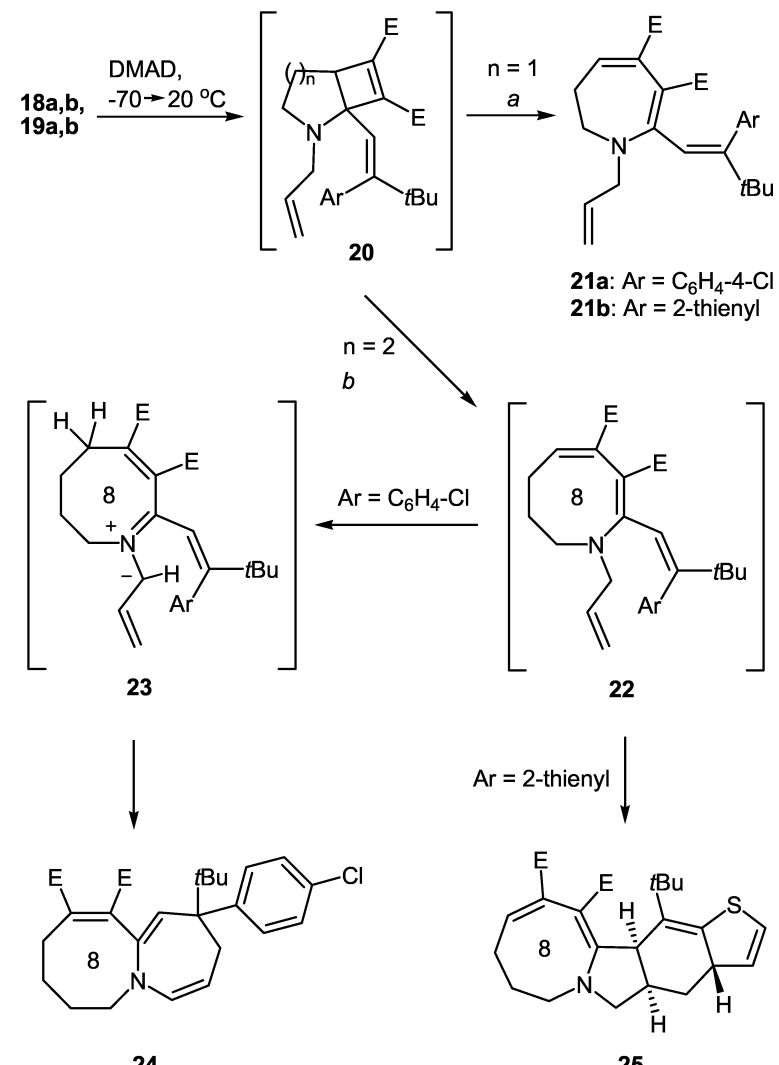

Scheme 8 . $6 \rightarrow 8$ Ring expansion of aminodienes 18 and 19 and subsequent thermal isomerization reactions $(\mathrm{E}=$ $\left.\mathrm{CO}_{2} \mathrm{Me}\right) ;(a)$ toluene, $120{ }^{\circ} \mathrm{C}, 5 \mathrm{~h} ;(b)$ toluene, $160{ }^{\circ} \mathrm{C}, 5 \mathrm{~h}$.

tra (see Experimental Section). It is obvious that $\mathbf{2 4}$ and $\mathbf{2 5}$ are derived from $N$-allylazocines by subsequent thermally induced isomerization. Conversion of monocyclic $\mathbf{2 2}$ into tetracyclic $\mathbf{2 5}$ occurs by an intramolecular Diels-Alder reaction with the 2-vinylthiophene unit as the diene component. If no suitable diene unit is present $(\mathrm{Ar}=4$-chlorophenyl), a [1,6] shift of an allylic $\mathrm{NCH}$ hydrogen atom generates the conjugated azomethine ylide 23 which yields bicyclic 24 by an $8 \pi$ 1,7-electrocyclic ring closure. 1,7-Electrocyclizations of conjugated 1,3-dipoles are an established route to seven-membered heterocycles [18].

In conclusion, semicyclic 2-amino-1,3-dienes in which the enamine function is incorporated in a five-, six-, or seven-membered ring were found to react with DMAD at the enamine $\mathrm{C}=\mathrm{C}$ bond to give two-carbon ring-expanded products, while a [4+2] cycloaddition was not observed. 6,7-Dihydro- $1 H$-azepines could be isolated in good yield and were thermally quite stable at high temperatures, surviving treatment at more 


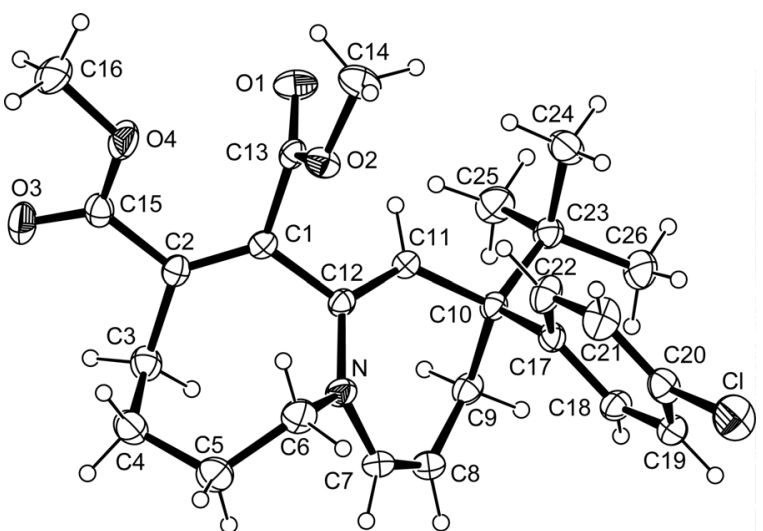

Fig. 3. Solid-state structure of $\mathbf{2 4}$; ellipsoids of thermal vibration are shown at the $30 \%$ probability level. Selected bond lengths $[\AA]$, bond angles $\left[{ }^{\circ}\right]$, and torsion angles $\left[{ }^{\circ}\right]$ : $\mathrm{C} 1-\mathrm{C} 2$ 1.342(2), C1-C13 1.504(3), C2-C15 1.494(3), C1-C12 $1.500(3), \mathrm{C} 11-\mathrm{C} 12$ 1.340(2), C7-C8 1.328(3); C6-N-C12 120.73(16), C6-N-C7 115.90(17), C7-N-C12 123.37(16); C13-C1-C2-C15 1.9(3), C10-C11-C12-N -3.4(3), C6$\mathrm{N}-\mathrm{C} 12-\mathrm{C} 1-32.0(2)$.

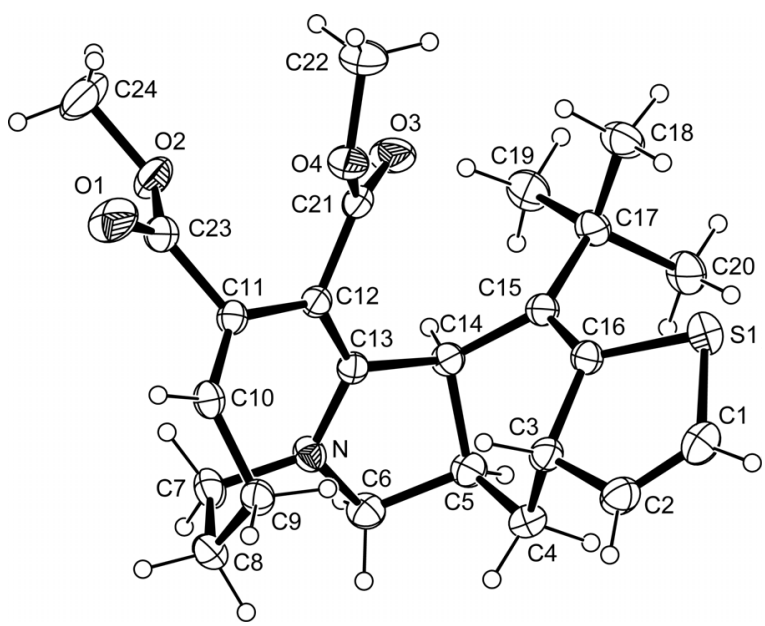

Fig. 4. Solid-state structure of 25; ellipsoids of thermal vibration are shown at the $30 \%$ probability level. Selected bond lengths $[\AA]$, bond angles $\left[{ }^{\circ}\right]$, and torsion angles $\left[{ }^{\circ}\right]$ : $\mathrm{C} 10-\mathrm{C} 11$ 1.338(2), C11-C12 1.488(2), C11-C23 1.500(2), C12-C21 1.462(2), C12-C13 1.380(2), C13-N 1.356(2), N-C6 1.458(2), C15-C16 1.337(3), C1-C2 1.315(3); C6-N-C7 117.46(15), C6-N-C13 114.21(14), $\mathrm{C} 7-\mathrm{N}-\mathrm{C} 13$ 127.75(15), C11-C12-C13-N 10.3(3), C10C11-C12-C13 39.4(3).

than $200{ }^{\circ} \mathrm{C}(\mathbf{3 a}-\mathbf{c})$ or at $120^{\circ} \mathrm{C}(\mathbf{2 1 a}, \mathbf{b})$. On the other hand, the homologous azocine and azonine derivatives were found to undergo a variety of thermally induced isomerization reactions, including transannular reaction as well as cyclization or cycloaddition reactions involving the exocyclic $N$-allyl and the adja- cent alkenyl substituents. The greater conformational flexibility of the eight- and nine-membered ring systems is likely to favor these thermal isomerization reactions. The results reported here indicate once more that the two-carbon ring expansion of endocyclic enamines is a suitable method to prepare functionalized seven-, eight-, and nine-membered azaheterocycles. The latter two classes in particular, representing typical mediumsized ring systems, have been studied relatively little and are found only in a limited number of natural products $[2,19]$. However, some azonine and azocine derivatives are known to have interesting pharmaceutical properties.

\section{Experimental Section}

General methods: Solvents were dried by standard methods and were stored under an argon atmosphere. The petroleum ether used had a boiling point range of $40-$ $60{ }^{\circ} \mathrm{C}$. Dimethyl acetylenedicarboxylate (DMAD) was distilled prior to use. All reactions involving aminodienes were carried out in rigorously dried glassware and under an argon atmosphere. Column chromatography was performed using hydrostatic pressure (silica gel 60, Macherey-Nagel, $0.063-$ $0.2 \mathrm{~mm}$ ) or under elevated pressure using Merck Lichroprep Si60 columns (particle size $40-63 \mu \mathrm{m}$, two columns of size A and B connected), a gradient pump (Merck-Hitachi L6200) and UV detection (Gilson Spectrochrom M, $\lambda=$ $254 \mathrm{~nm}$ ). For the bulb-to-bulb distillation experiments, the temperature of the heating mantle is given. The NMR spectra were recorded in $\mathrm{CDCl}_{3}$ solution using as the internal standard tetramethylsilane for ${ }^{1} \mathrm{H}$ spectra $(\delta=0 \mathrm{ppm})$ and residual solvent signal $\left(\delta\left(\mathrm{CHCl}_{3}\right)=77.0 \mathrm{ppm}\right)$ for ${ }^{13} \mathrm{C}$ spectra; $\mathrm{mc}=$ centered multiplet. The signal assignment was based on proton-coupled ${ }^{13} \mathrm{C}$ spectra $\left({ }^{1} J(\mathrm{C}, \mathrm{H})\right.$ multiplicities are given with the spectra), $\mathrm{H}, \mathrm{H}$ COSY, and $\mathrm{C}, \mathrm{H}\left({ }^{1} \mathrm{~J}\right)$ as well as $\mathrm{C}, \mathrm{H}\left({ }^{2} J,{ }^{3} J\right)$ correlation spectra. IR spectra were recorded on Perkin Elmer IR 883 and IR 1310 spectrometers. Microanalyses were carried out in the Analytical Laboratories of the Universities of Ulm and Kaiserslautern.

Dimethyl 2-[(Z)-2-(4-chlorophenyl)-3,3-dimethylbut-1enyl]-6,7-dihydro-1-methyl-1H-azepine-3,4-dicarboxylate (3a): A solution of aminodiene 1a [6] $(0.83 \mathrm{~g}, 3.0 \mathrm{mmol})$ in ether $(10 \mathrm{ml})$ was cooled at $-40{ }^{\circ} \mathrm{C}$ and DMAD $(0.41 \mathrm{ml}$, $3.3 \mathrm{~mol}$ ) was added. The cooling bath was removed and the mixture was stirred overnight. After removal of the solvent at $0.01 \mathrm{mbar}$, the residual oil was submitted to a Kugelrohr distillation. Excess DMAD was distilled off at $120{ }^{\circ} \mathrm{C} / 0.005$ mbar. The temperature was raised until a yellow oil distilled over at $220{ }^{\circ} \mathrm{C} / 0.005$ mbar. It was dissolved in ether, and pentane was added until the solution started to become turbid. Crystallization at $-30{ }^{\circ} \mathrm{C}$ furnished yellow crystals $(0.56 \mathrm{~g}, 45 \%)$, m.p. $93{ }^{\circ} \mathrm{C}$. - 
IR $(\mathrm{KBr}): v=2940(\mathrm{~m}), 1730-1630(\mathrm{~s}$, broad $) \mathrm{cm}^{-1}$. ${ }^{1} \mathrm{H}$ NMR (400.13 MHz): $\delta=1.16(\mathrm{~s}, 9 \mathrm{H}, t \mathrm{Bu}), 1.80-2.15$ (m, br, $\left.2 \mathrm{H}, 6-\mathrm{H}_{2}\right), 2.67$ (s, $\left.3 \mathrm{H}, \mathrm{NCH}_{3}\right), 2.60-2.85$ (m, $\left.2 \mathrm{H}, \mathrm{NCH}_{2}\right), 3.65\left(\mathrm{~s}, 3 \mathrm{H}, \mathrm{OCH}_{3}\right), 3.69\left(\mathrm{~s}, 3 \mathrm{H}, \mathrm{OCH}_{3}\right)$, $6.52(\mathrm{t}, 1 \mathrm{H}, J=6.1 \mathrm{~Hz}, 5-\mathrm{H}), 6.54\left(\mathrm{~s}, 1 \mathrm{H},=\mathrm{CH}_{\text {excycl. }}\right)$, 6.94/7.26 (AA'BB', $4 \mathrm{H}_{\text {aryl }}$ ). - ${ }^{13} \mathrm{C}$ NMR (100.61 MHz): $\delta=27.7(\mathrm{C}-6), 29.2\left(\mathrm{CMe}_{3}\right), 36.3\left(\mathrm{CMe}_{3}\right), 41.1\left(\mathrm{NCH}_{3}\right)$, $50.9\left(\mathrm{OCH}_{3}\right), 51.9\left(\mathrm{OCH}_{3}\right), 57.7(\mathrm{C}-7), 99.7(\mathrm{~s}, \mathrm{NC}=C)$, 124.8 (d), 127.3 (d), 129.7 (d), 132.6 (s), 133.5 (s), 135.0 (d, C-5), 138.2 (s), 151.2 (s), 157.9 (s), 168.9 (C=O), 169.9 $(\mathrm{C}=\mathrm{O}) .-\mathrm{C}_{23} \mathrm{H}_{28} \mathrm{ClNO}_{4}$ (417.93): calcd. C 66.10, H 6.75, N 3.35; found C 66.1, H 6.8, N 3.3.

Dimethyl 2-[(Z)-2-(furan-2-yl)-3,3-dimethylbut-1-enyl]-6,7-dihydro-1-methyl-1H-azepine-3,4-dicarboxylate

(3b): Prepared as described for $\mathbf{3 a}$, from aminodiene $\mathbf{1 b}$ [6] $(0.70 \mathrm{~g}, 3.0 \mathrm{mmol})$ and DMAD (0.41 ml, $3.3 \mathrm{mmol})$. Kugelrohr distillation at $200{ }^{\circ} \mathrm{C} / 0.005$ mbar. Yellow microcrystalline solid $(0.59 \mathrm{~g}, 53 \%)$, m.p. $84{ }^{\circ} \mathrm{C}$. - IR ( $\left.\mathrm{KBr}\right)$ : $v=3100$ (w), 2940 (s), 1710 (s), 1685 (s) $\mathrm{cm}^{-1}$. - ${ }^{1} \mathrm{H}$ NMR (200.1 MHz): $\delta=1.22$ (s, $9 \mathrm{H}, t \mathrm{Bu}), 2.36$ (virtual q, $2 \mathrm{H}$, 6- $\left.\mathrm{H}_{2}\right), 2.62$ (s, $\left.3 \mathrm{H}, \mathrm{NCH}_{3}\right), 2.99\left(\mathrm{~m}, 2 \mathrm{H}, \mathrm{NCH}_{2}\right), 3.62$ (s, $\left.3 \mathrm{H}_{1} \mathrm{OCH}_{3}\right), 3.71\left(\mathrm{~s}, 3 \mathrm{H}, \mathrm{OCH}_{3}\right), 6.12(\mathrm{dd}, J=3.2,0.6 \mathrm{~Hz}$, $\left.1 \mathrm{H}, 3-\mathrm{H}_{\text {furyl }}\right), 6.32\left(\mathrm{mc}, 1 \mathrm{H}, 4-\mathrm{H}_{\text {furyl }}\right), 6.53(\mathrm{t}, J=5.7 \mathrm{~Hz}$, $1 \mathrm{H}, 5-\mathrm{H}), 6.58\left(\mathrm{~s}, 1 \mathrm{H},=\mathrm{CH}_{\text {excycl. }}\right), 7.37(\mathrm{dd}, J=1.7,0.6 \mathrm{~Hz}$, $\left.1 \mathrm{H}, 5-\mathrm{H}_{\text {furyl }}\right) .-{ }^{13} \mathrm{C}$ NMR (100.61 MHz): $\delta=28.5(\mathrm{C}-6)$, $29.1\left(\mathrm{CMe}_{3}\right), 36.4\left(\mathrm{CMe}_{3}\right), 41.0\left(\mathrm{NCH}_{3}\right), 50.5\left(\mathrm{OCH}_{3}\right)$, $51.6\left(\mathrm{OCH}_{3}\right), 57.7(\mathrm{C}-7), 98.9(\mathrm{~s}, \mathrm{NC}=C), 109.5(\mathrm{~d})$, 110.0 (d), 126.5 (d), 133.5 (d, $J=160.0 \mathrm{~Hz}, \mathrm{C}-5), 141.3$ $\left(\mathrm{d}, J=211.3 \mathrm{~Hz}, 5-\mathrm{C}_{\text {furyl }}\right), 143.6$ (s), $152.3(\mathrm{~s}), 158.5$ (s), $168.7(\mathrm{C}=\mathrm{O}), 170.2(\mathrm{C}=\mathrm{O}) .-\mathrm{C}_{21} \mathrm{H}_{27} \mathrm{NO}_{5}$ (373.45): calcd. C 67.54, H 7.29; N 3.75; found C 67.2, H 7.2, N 3.7.

Dimethyl 6,7-dihydro-1-methyl-2-[(Z)-3,3-dimethyl-2(thiophen-2-yl)but-1-enyl]-1H-azepine-3,4-dicarboxylate

(3c): Prepared as described for $\mathbf{3 a}$, from aminodiene 1c [6] $(0.74 \mathrm{~g}, 3.0 \mathrm{mmol})$ and DMAD $(0.41 \mathrm{ml}, 3.3 \mathrm{mmol})$; Kugelrohr distillation at $240{ }^{\circ} \mathrm{C} / 0.005$ mbar. Yellow crystals $(0.76$ g, $65 \%)$, m.p. $72{ }^{\circ} \mathrm{C}$. - IR (KBr): $v=3100$ (w), $2940(\mathrm{~m}), 1710(\mathrm{~s}), 1680(\mathrm{~s}), 1625(\mathrm{~m}) \mathrm{cm}^{-1} .-{ }^{1} \mathrm{H}$ NMR (400.13 MHz): $\delta=1.22(\mathrm{~s}, 9 \mathrm{H}, t \mathrm{Bu}), 2.0-2.2(\mathrm{~m}, 2 \mathrm{H}$, 6- $\mathrm{H}_{2}$ ), 2.69 (s, $3 \mathrm{H}, \mathrm{NCH}_{3}$ ), 2.7-3.1 (unresolved m, $2 \mathrm{H}$, $\left.\mathrm{NCH}_{2}\right), 3.62\left(\mathrm{~s}, 3 \mathrm{H}, \mathrm{OCH}_{3}\right), 3.68\left(\mathrm{~s}, 3 \mathrm{H}, \mathrm{OCH}_{3}\right), 6.55$ $(\mathrm{t}, J=6.0 \mathrm{~Hz}, 1 \mathrm{H}, 5-\mathrm{H}), 6.62\left(\mathrm{~s}, 1 \mathrm{H},=\mathrm{CH}_{\text {exocycl. }}\right), 6.73$ (dd, $\left.J=3.4,1.2 \mathrm{~Hz}, 1 \mathrm{H}, 3-\mathrm{H}_{\text {thienyl }}\right), 6.94(\mathrm{mc}, 1 \mathrm{H}$, $\left.4-\mathrm{H}_{\text {thienyl }}\right), 7.21\left(\mathrm{dd}, J=5.1,1.1 \mathrm{~Hz}, 5-\mathrm{H}_{\text {thienyl }}\right) \cdot-{ }^{13} \mathrm{C} \mathrm{NMR}$ (100.61 MHz): $\delta=27.6(\mathrm{C}-6), 28.8\left(\mathrm{CMe}_{3}\right), 36.0\left(\mathrm{CMe}_{3}\right)$, $40.7\left(\mathrm{NCH}_{3}\right), 50.3\left(\mathrm{OCH}_{3}\right), 51.3\left(\mathrm{OCH}_{3}\right), 57.8(\mathrm{C}-7)$, 99.4 (s, NC=C), 124.1 (d), 125.6 (d), 125.7 (d), 126.9 (d), 133.6 (s), 134.6 (d, $J=160.1 \mathrm{~Hz}, \mathrm{C}-5), 138.9$ (s), 154.3 (s), 157.4 (s), $168.4(\mathrm{C}=\mathrm{O}), 169.4(\mathrm{C}=\mathrm{O}) . \quad-\mathrm{C}_{21} \mathrm{H}_{27} \mathrm{NO}_{4} \mathrm{~S}$ (389.51): calcd. C 64.75, H 6.99, N 3.60; found C 64.5, H 7.0, N 3.5.

Dimethyl 2-\{2-[(Z)-2-(4-chlorophenyl)-3,3-dimethylbut-1 -enyl]-4,5-dihydro-1-methyl-1H-pyrrol-3-yl\}maleate (or fu- marate) (4a): The reaction of aminodiene 1a (0.83 g, $3.0 \mathrm{mmol})$ and DMAD $(0.41 \mathrm{ml}, 3.3 \mathrm{mmol})$ was conducted as described above for 3a. After stirring overnight, the solvent was evaporated at $25{ }^{\circ} \mathrm{C} / 0.01 \mathrm{mbar}$, and the residue was worked up by column chromatography (silica gel, ether/ $\mathrm{CH}_{2} \mathrm{Cl}_{2}(1: 1)$ ). Crystallization from ether yielded $\mathbf{4 a}$ as a yellow powder $(0.95 \mathrm{~g}, 76 \%)$, m. p. $103{ }^{\circ} \mathrm{C}$. $-\mathrm{IR}(\mathrm{KBr})$ : $v=2970$ (s), 2945 (s), 2920 (s), 2900 (s), 1725 (vs), 1685 (s) $\mathrm{cm}^{-1} .-{ }^{1} \mathrm{H}$ NMR $(200.1 \mathrm{MHz}): \delta=1.15(\mathrm{~s}, 9 \mathrm{H}, t \mathrm{Bu})$, 2.25 - 2.50 (unresolved $\mathrm{m}, 2 \mathrm{H}$ ), $2.64\left(\mathrm{~s}, 3 \mathrm{H}, \mathrm{NCH}_{3}\right), 3.10$ (t, $\left.2 \mathrm{H}, \mathrm{NCH}_{2}\right), 3.66\left(\mathrm{~s}, 3 \mathrm{H}, \mathrm{OCH}_{3}\right), 3.95\left(\mathrm{~s}, 3 \mathrm{H}, \mathrm{OCH}_{3}\right)$, $4.92\left(\mathrm{~s}, 1 \mathrm{H},=\mathrm{C} H-\mathrm{CO}_{2} \mathrm{Me}, 5.92(\mathrm{~s}, 1 \mathrm{H}, \mathrm{CH}=\mathrm{C}-\mathrm{Aryl})\right.$, 7.11/7.21 (AA'BB', $4 \mathrm{H}_{\text {aryl }}$ ). $-{ }^{13} \mathrm{C}$ NMR (100.61 MHz): $\delta=$ $28.4\left(\mathrm{NCH}_{2} \mathrm{CH}_{2}\right), 29.2\left(\mathrm{CMe}_{3}\right), 35.1\left(\mathrm{NCH}_{3}\right), 36.9\left(\mathrm{CMe}_{3}\right)$, $50.8\left(\mathrm{OCH}_{3}\right), 52.2\left(\mathrm{NCH}_{2}\right), 52.6\left(\mathrm{OCH}_{3}\right), 101.3(\mathrm{CH}-$ $\mathrm{CO}_{2} \mathrm{Me}$ ), 105.6 (s, $\left.\mathrm{NC}=C\right), 113.7(\mathrm{CH}=\mathrm{C}-\mathrm{Aryl}), 127.3(\mathrm{~d})$, 130.0 (d), 132.9 (s), 136.9 (s), $146.0 \mathrm{~s}), 155.7$ (s), 158.8 (s), $167.6(\mathrm{C}=\mathrm{O}), 169.8(\mathrm{C}=\mathrm{O}) .-\mathrm{C}_{23} \mathrm{H}_{28} \mathrm{ClNO}_{4}$ (417.93): calcd. C 66.10, H 6.75, N 3.35; found C 65.9, H 6.8, N 3.3.

Dimethyl 2-\{2-[(Z)-2-(2-furanyl)-3,3-dimethylbut-1-enyl]-4,5-dihydro-1-methyl-1H-pyrrol-3-yl $\}$ maleate (or fumarate) (4b): The reaction of aminodiene 1b [6] (0.69 g, 3.0 $\mathrm{mmol})$ and DMAD $(0.41 \mathrm{ml}, 3.3 \mathrm{mmol})$ was conducted as described above for 3a. After stirring overnight, the solvent was evaporated at $25{ }^{\circ} \mathrm{C} / 0.01 \mathrm{mbar}$, and the residue was worked up by column chromatography (silica gel, ether/ $\mathrm{CH}_{2} \mathrm{Cl}_{2}$ (1:1)). Crystallization from ether yielded $\mathbf{4 b}$ as light-red crystals $\left(0.92\right.$ g, 82\%), m.p. $164{ }^{\circ} \mathrm{C}$. - IR (KBr): $v=$ 2960 (m), 2900 (m), 1715 (vs), 1670 (s) $\mathrm{cm}^{-1} .-{ }^{1} \mathrm{H}$ NMR (400.13 MHz): $\delta=1.26(\mathrm{~s}, 9 \mathrm{H}, t \mathrm{Bu}), 2.42\left(\mathrm{~s}, 3 \mathrm{H}, \mathrm{NCH}_{3}\right)$, $2.61(\mathrm{mc}, 2 \mathrm{H}), 3.20-3.40$ (unresolved $\mathrm{m}, 2 \mathrm{H}), 3.65$ (s, $3 \mathrm{H}$, $\left.\mathrm{OCH}_{3}\right), 3.84\left(\mathrm{~s}, 3 \mathrm{H}, \mathrm{OCH}_{3}\right), 4.99\left(\mathrm{~s}, 1 \mathrm{H},=\mathrm{CH}-\mathrm{CO}_{2} \mathrm{Me}\right)$, $5.92(\mathrm{~s}, 1 \mathrm{H}, \mathrm{C} H=\mathrm{C}-\mathrm{Aryl}), 6.32(\mathrm{dd}, J=3.3,1.7 \mathrm{~Hz}, 1 \mathrm{H}$, $\left.4-\mathrm{H}_{\text {furyl }}\right), 6.42\left(\mathrm{dd}, J=3.3,0.7 \mathrm{~Hz}, 1 \mathrm{H}, 3-\mathrm{H}_{\text {furyl }}\right), 7.38$ (dd, $\left.1 \mathrm{H}, J=1.8,0.8 \mathrm{~Hz}, 5-\mathrm{H}_{\text {furyl }}\right) .-{ }^{13} \mathrm{C} \mathrm{NMR}(100.61 \mathrm{MHz})$ : $\delta=28.2\left(\mathrm{NCH}_{2} \mathrm{CH}_{2}\right), 29.4\left(\mathrm{CMe}_{3}\right), 33.9\left(\mathrm{NCH}_{3}\right), 36.9$ $\left(\mathrm{CMe}_{3}\right), 50.6\left(\mathrm{OCH}_{3}\right), 52.1\left(\mathrm{OCH}_{3}\right), 52.7\left(\mathrm{NCH}_{2}\right), 100.4$ $\left(=C \mathrm{H}-\mathrm{CO}_{2} \mathrm{Me}\right), 105.8(\mathrm{~s}, \mathrm{NC}=C), 110.6(\mathrm{~d}), 111.4(\mathrm{~d}), 115.1$ (CH=C-Aryl), 141.6 (d, $\left.J=201.8 \mathrm{~Hz}, \mathrm{C}-5_{\text {furyl }}\right), 146.2$ (s), $149.5(\mathrm{~s}), 151.6(\mathrm{~s}), 155.7(\mathrm{~s}), 167.6(\mathrm{C}=\mathrm{O}), 169.8(\mathrm{C}=\mathrm{O})$. $\mathrm{C}_{21} \mathrm{H}_{27} \mathrm{NO}_{5}$ (373.45): calcd. C 67.54, H 7.29, N 3.75; found C 67.0, H 7.4, N 3.6.

Dimethyl 2-[(Z)-2-(furan-2-yl)-3,3-dimethylbut-1-enyl]6,7-dihydro-1-methyl-1H-azepine-3,4-dicarboxylate (8):

(2E)-2-[2-(Furan-2-yl)prop-2-en-1-ylidene]-2,3,4,5-tetrahydro-1-methyl-1H-pyrrole (7) was prepared according to a procedure for similar aminodienes [6] and was used without purification. The reaction of crude aminodiene $7(0.57 \mathrm{~g}$, $3.0 \mathrm{mmol})$ and DMAD $(0.41 \mathrm{ml}, 3.3 \mathrm{mmol})$ was carried out as described above for 3a. Bulb-to-bulb distillation at $200{ }^{\circ} \mathrm{C} / 0.005$ mbar followed by crystallization from ether/pentane furnished a yellow, microcrystalline solid $(0.58$ g, $58 \%)$, m. p. $93{ }^{\circ} \mathrm{C}$. - IR (KBr): $v=2950$ (s), 1710 
(sh), 1695 (s) cm ${ }^{-1}$. - ${ }^{1} \mathrm{H}$ NMR (200.1 MHz): $\delta=2.20$ (d, $\left.{ }^{4} J=1.1 \mathrm{~Hz}, 3 \mathrm{H},=\mathrm{C}-\mathrm{CH}_{3}\right), 2.61(\mathrm{mc}, 2 \mathrm{H}, 6-\mathrm{H}), 2.88(\mathrm{~s}$, $\left.3 \mathrm{H}, \mathrm{NCH}_{3}\right), 3.40$ (t, $\left.2 \mathrm{H}, \mathrm{NCH}_{2}\right), 3.54\left(\mathrm{~s}, 3 \mathrm{H}, \mathrm{OCH}_{3}\right), 3.72$ (s, $3 \mathrm{H}, \mathrm{OCH}_{3}$ ), 6.41 (mc, $\left.1 \mathrm{H}\right), 6.42$ ("s", ${ }^{4} J$ coupling not resolved, $\left.1 \mathrm{H},=\mathrm{CH}_{\text {exocycl. }}\right), 6.50(\mathrm{t}, 1 \mathrm{H}, J=5.4 \mathrm{~Hz}, 5-\mathrm{H}), 6.84$ (mc, $1 \mathrm{H}), 7.41(\mathrm{mc}, 1 \mathrm{H}) .-{ }^{13} \mathrm{C} \mathrm{NMR}(100.61 \mathrm{MHz}): \delta=$ $14.3\left(=\mathrm{C}-\mathrm{CH}_{3}\right), 29.2(\mathrm{C}-6), 40.7\left(\mathrm{NCH}_{3}\right), 50.7\left(\mathrm{OCH}_{3}\right), 51.8$ $\left(\mathrm{OCH}_{3}\right), 55.6(\mathrm{C}-7), 98.5(\mathrm{~s}, \mathrm{NC}=C), 107.6(\mathrm{~d}), 111.2$ (d), 121.5 (d), 131.0 (s), 131.9 (d, $J=156.4 \mathrm{~Hz}, \mathrm{C}-5), 133.4$ (s), 142.4 (d, $\left.J=202.4 \mathrm{~Hz}, \mathrm{C}-5_{\text {furyl }}\right) 154.7$ (s), 157.5 (s), 168.9 $(\mathrm{C}=\mathrm{O}), 170.8(\mathrm{C}=\mathrm{O}) .-\mathrm{C}_{18} \mathrm{H}_{21} \mathrm{NO}_{5}$ (331.37): calcd. $\mathrm{C}$ 65.24, H 6.39, N 4.23; found C 64.0, H 6.7, N 3.8.

Dimethyl 6,7,8,9-tetrahydro-1-methyl-2-[(thiophen-2-yl) ethynyl]-1H-azonine-3,4-dicarboxylate (10): A solution of alkynyl-enamine 9 [10] $(0.65 \mathrm{~g}, 3.0 \mathrm{mmol})$ in ether $(10 \mathrm{ml})$ was cooled at $-40{ }^{\circ} \mathrm{C}$ and DMAD $(0.41 \mathrm{ml}, 3.3 \mathrm{mmol})$ was added dropwise. The cooling bath was removed and the solution was stirred overnight. After evaporation of the solvent at $25^{\circ} \mathrm{C} / 0.01 \mathrm{mbar}$, the residue was extracted with ether $(4 \times 50 \mathrm{ml})$. The extracts were combined, the solvent was removed, and the residue was crystallized by addition of $\mathrm{CH}_{2} \mathrm{Cl}_{2}$ /ether and cooling at $-30^{\circ} \mathrm{C}$. Yellow crystals $(0.89 \mathrm{~g}$, 83\%), m.p. $114{ }^{\circ} \mathrm{C}$. - IR (KBr): $v=3060$ (w), 2920 (s), 2180 (s, C $\equiv \mathrm{C}$ ), 1700 (vs), 1670 (vs) $\mathrm{cm}^{-1} .-{ }^{1} \mathrm{H}$ NMR (400.13 MHz): $\delta=1.60-1.88(\mathrm{~m}, 4 \mathrm{H}), 2.26(\mathrm{mc}, 2 \mathrm{H})$, 3.03 (s, $\left.3 \mathrm{H}, \mathrm{NCH}_{3}\right), 3.34\left(\mathrm{mc}, 2 \mathrm{H}, \mathrm{NCH}_{2}\right), 3.68(\mathrm{~s}, 3 \mathrm{H}$, $\left.\mathrm{OCH}_{3}\right), 3.73\left(\mathrm{~s}, 3 \mathrm{H}, \mathrm{OCH}_{3}\right), 6.98(\mathrm{t}, J=9.2 \mathrm{~Hz}, 1 \mathrm{H}, 5-$ $\mathrm{H}), 7.02\left(\mathrm{dd}, J=5.2,3.8 \mathrm{~Hz}, 1 \mathrm{H}, 4-\mathrm{H}_{\text {thienyl }}\right), 7.33$ (dd, $\left.J=3.5,1.1 \mathrm{~Hz}, 1 \mathrm{H}, 3-\mathrm{H}_{\text {thienyl }}\right), 7.37(\mathrm{dd}, J=5.2,1.1 \mathrm{~Hz}$, $\left.1 \mathrm{H}, 5-\mathrm{H}_{\text {thienyl }}\right) .-{ }^{13} \mathrm{C} \mathrm{NMR}(100.61 \mathrm{MHz}): \delta=22.7\left(\mathrm{CH}_{2}\right)$, $24.9\left(\mathrm{CH}_{2}\right), 27.5\left(\mathrm{CH}_{2}\right), 41.1\left(\mathrm{NCH}_{3}\right), 49.8\left(\mathrm{CH}_{2}\right), 51.1$ $\left(\mathrm{OCH}_{3}\right), 51.8\left(\mathrm{OCH}_{3}\right), 88.4$ and $91.7(\mathrm{C} \equiv \mathrm{C}), 104.4(\mathrm{~s}, \mathrm{C}-3)$, 122.0 (s), 127.1 (d), 128.7 (d), 132.4 (s), 132.9 (d), 142.0 (d, $J=156.8 \mathrm{~Hz}, \mathrm{C}-5), 142.2(\mathrm{~s}), 168.0(\mathrm{C}=\mathrm{O}), 168.2(\mathrm{C}=\mathrm{O})$. $\mathrm{C}_{19} \mathrm{H}_{21} \mathrm{NO}_{4} \mathrm{~S}$ : calcd. C 63.49, H 5.89, N 3.90; found C 63.5, H 5.8, N 3.9.

Dimethyl (1(2')E,2E,4Z)- and (1(2')E,2E,4E)-1-(1-methylpiperidin-2-ylidene)-5-(thiophen-2-yl)hexa-2,4-diene-1,2dicarboxylate (14): A solution of aminodiene 11/12 [6] $(0.70 \mathrm{~g}, 3.0 \mathrm{mmol})$ in ether $(10 \mathrm{ml})$ was cooled at $-40{ }^{\circ} \mathrm{C}$ and DMAD (0.41 ml, $3.3 \mathrm{mmol})$ was added dropwise. The solution was brought to r.t. and submitted to a Kugelrohr distillation. Excess DMAD was distilled off at 100$120{ }^{\circ} \mathrm{C} / 0.005$ mbar. At $230{ }^{\circ} \mathrm{C} / 0.005 \mathrm{mbar}$, a red oil distilled over from which orange crystals were obtained after crystallization from ether/pentane. Yield: $0.69 \mathrm{~g}(61 \%)$; mixture of diastereomers, (1(2')E,2E,4Z):(1(2')E,2E,4E) = 3:1. M.p. $119{ }^{\circ} \mathrm{C}$. - IR (KBr): $v=2930$ (m), 1670 (s, br) $\mathrm{cm}^{-1}$. - ${ }^{1} \mathrm{H}$ NMR (400.13 MHz) of $\left(1\left(2^{\prime}\right) E, 2 E, 4 Z\right)-14$ : $\delta=1.72-1.84\left(\mathrm{~m}, 4 \mathrm{H}, \mathrm{NCH}_{2} \mathrm{CH}_{2} \mathrm{CH}_{2}\right), 2.30(\mathrm{~s}, 3 \mathrm{H}$, $\left.=\mathrm{C}_{-} \mathrm{CH}_{3}\right), 2.56\left(\mathrm{~s}, 3 \mathrm{H}, \mathrm{NCH}_{3}\right), 3.06-3.19(\mathrm{~m}, 4 \mathrm{H}), 3.59$ $\left(\mathrm{s}, 3 \mathrm{H}, \mathrm{OCH}_{3}\right), 3.75\left(\mathrm{~s}, 3 \mathrm{H}, \mathrm{OCH}_{3}\right), 6.27$ (broadened d, ${ }^{3} J=11.6 \mathrm{~Hz}, \mathrm{C} H=\mathrm{C}-$ Thienyl), $6.99(\mathrm{dd}, J=5.1,3.7 \mathrm{~Hz}$, $\left.1 \mathrm{H}, 4-\mathrm{H}_{\text {thienyl }}\right), 7.11\left(\mathrm{~d}, J=3.6 \mathrm{~Hz}, 1 \mathrm{H}, 3-\mathrm{H}_{\text {thienyl }}\right), 7.21$ $\left(\mathrm{d}, J=5.1 \mathrm{~Hz}, 1 \mathrm{H}, 5-\mathrm{H}_{\text {thienyl }}\right), 7.51(\mathrm{~d}, J=11.6 \mathrm{~Hz}, 1 \mathrm{H}$, ester-C=CH); $\left(1\left(2^{\prime}\right) E, 2 E, 4 E\right)-14: \delta=6.62(\mathrm{~d}, J=11.6 \mathrm{~Hz}$, $\mathrm{CH}=\mathrm{C}-$ Thienyl). $-{ }^{13} \mathrm{C}(100.61 \mathrm{MHz}): \delta=16.1\left(=\mathrm{C}-\mathrm{CH}_{3}\right)$, $20.5\left(\mathrm{CH}_{2}\right), 22.8\left(\mathrm{CH}_{2}\right), 29.8\left(\mathrm{CH}_{2}\right), 44.4\left(\mathrm{NCH}_{3}\right), 50.5$ $\left(\mathrm{OCH}_{3}\right), 51.4\left(\mathrm{CH}_{2}\right), 51.7\left(\mathrm{OCH}_{3}\right), 93.0(\mathrm{~s}, \mathrm{NC}=\mathrm{C}), 121.3$ (d, $\mathrm{CH}=\mathrm{C}$-Thienyl), 124.4 (d), 125.0 (d), 127.5 (d), 130.2 (d, ester- $\mathrm{C}=\mathrm{CH}), 131.3(\mathrm{~s}), 135.7(\mathrm{~s}), 146.5(\mathrm{~s}), 164.1$ (s, $\mathrm{NC}=)$, $169.36(\mathrm{C}=\mathrm{O}), 169.40(\mathrm{C}=\mathrm{O}) .-\mathrm{C}_{20} \mathrm{H}_{25} \mathrm{NO}_{4} \mathrm{~S}$ (375.49): calcd. C 63.98, H 6.71, N 3.73; found C 64.0, H 6.8, N 3.9.

1-Allyl-5-[(Z)-2-(4-chlorophenyl)-3,3-dimethylbut-1-enyl]-2,3-dihydro-1H-pyrrole (18a): To a stirred suspension of copper(I) bromide dimethylsulfide $(0.432 \mathrm{~g}, 2.1 \mathrm{mmol})$ in THF $(10 \mathrm{ml})$, cooled at $-70{ }^{\circ} \mathrm{C}$, was slowly added a $1.7 \mathrm{M}$ solution of tert-butyl lithium in hexane $(2.37 \mathrm{ml}$, $4.2 \mathrm{mmol}$ ). The yellow suspension was then brought at $-40{ }^{\circ} \mathrm{C}$ within $10 \mathrm{~min}$ and kept at this temperature until a homogeneous pale-yellow solution had formed which was cooled again at $-70{ }^{\circ} \mathrm{C}$. A solution of iminium salt 16a [17] $(0.788 \mathrm{~g}, 2.0 \mathrm{mmol})$ in THF $(10 \mathrm{ml})$ was added dropwise. The mixture was warmed at $-40{ }^{\circ} \mathrm{C}$ within $15 \mathrm{~min}$ and kept at this temperature for $1 \mathrm{~h}$, then brought to r.t. within $2 \mathrm{~h}$, whereupon a black color appeared. The solvent was evaporated at $0.01 \mathrm{mbar}$ and the residue was extracted with pentane $(3 \times 50 \mathrm{ml})$. The extracts were combined and the solvent was completely evaporated at 0.01 mbar to leave a yellow oil which could not be purified further and was used directly (vide infra). Yield of crude 18a: $0.520 \mathrm{~g}$ (1.72 mmol, 86\%). - IR (film): $v=2960$ (m), 2867 (m), 1643 (m), 1487 (m), $1171(\mathrm{~m}), 1091(\mathrm{~m}) \mathrm{cm}^{-1} .-{ }^{1} \mathrm{H}$ NMR (500.14 MHz): $\delta=1.09$ (s, $9 \mathrm{H}, t \mathrm{Bu}), 2.19\left(\mathrm{mc}, 2 \mathrm{H}, 3-\mathrm{H}_{2}\right)$, $2.87\left(\mathrm{t}, 2 \mathrm{H}, 2-\mathrm{H}_{2}\right), 3.40\left(\mathrm{~d}, 2 \mathrm{H}, \mathrm{NCH}_{2} \mathrm{CH}=\right), 3.91(\mathrm{mc}, 1 \mathrm{H}$, $4-\mathrm{H}), 5.12\left(\mathrm{dd},{ }^{3} \mathrm{~J}=10.2,{ }^{2} \mathrm{~J}=1.6 \mathrm{~Hz}, 1 \mathrm{H}, \mathrm{CH}_{2} \mathrm{CH}=\mathrm{CH}_{2}\right.$ ), $5.19\left(\mathrm{dd},{ }^{3} J=17.2,{ }^{2} J=1.6 \mathrm{~Hz}, 1 \mathrm{H}, \mathrm{CH}_{2} \mathrm{CH}=\mathrm{CH}_{2}\right)$, $5.83\left(\mathrm{mc}, 1 \mathrm{H}, \mathrm{CH}_{2} \mathrm{CH}=\mathrm{CH}_{2}\right), 5.95(\mathrm{~s}, 1 \mathrm{H}, 5-\mathrm{CH}=)$, 6.95/7.26 (AA'BB', $4 \mathrm{H}_{\text {aryl }}$ ). $-{ }^{13} \mathrm{C} \mathrm{NMR}$ (125.77 MHz): $\delta=29.0(\mathrm{C}-3), 29.6\left(\mathrm{CMe}_{3}\right), 36.7\left(\mathrm{CMe}_{3}\right), 52.4(\mathrm{C}-2)$, $55.3\left(\mathrm{NCH}_{2} \mathrm{CH}=\right), 104.9$ (C-4), $115.4(\mathrm{CH}=\mathrm{C}-\mathrm{Aryl}), 116.8$ $\left(\mathrm{CH}_{2} \mathrm{CH}=\mathrm{CH}_{2}\right), 127.7$ (d), 130.9 (d), 132.2 (s, C-Cl), 135.8 $\left(\mathrm{CH}_{2} \mathrm{CH}=\mathrm{CH}_{2}\right), 139.0$ (s), $147.6(\mathrm{CH}=\mathrm{C}-\mathrm{Aryl}), 153.0$ (s, C-5). $-\mathrm{C}_{19} \mathrm{H}_{24} \mathrm{ClN}$ (301.86).

1-Allyl-5-[(Z)-3,3-dimethyl-2-(thiophen-2-yl)but-1-enyl]2,3-dihydro-1H-pyrrole (18b): The synthesis was achieved as described for $18 \mathbf{a}$, from a solution of $(t-\mathrm{Bu})_{2} \mathrm{CuLi} \cdot \mathrm{LiBr}$ $(4.2 \mathrm{mmol})$ in THF $(10 \mathrm{ml})$ and a solution of iminium salt 16b [17] $(0.731 \mathrm{~g}, 2.0 \mathrm{mmol})$ in THF $(10 \mathrm{ml})$. An orange oil was obtained which could not be purified further and was used directly (vide infra). Yield of crude 18b: $0.494 \mathrm{~g}$ (90\%). - IR (film): $v=3070$ (w), 2962 (m), $2865(\mathrm{~m}), 1613(\mathrm{~m}), 1462(\mathrm{~m}), 1224(\mathrm{~m}) \mathrm{cm}^{-1} .-{ }^{1} \mathrm{H}$ NMR (500.14 MHz): $\delta=1.14(\mathrm{~s}, 9 \mathrm{H}, t \mathrm{Bu}), 2.20\left(\mathrm{t}, 2 \mathrm{H}, 3-\mathrm{H}_{2}\right)$, $2.90\left(\mathrm{t}, 2 \mathrm{H}, 2-\mathrm{H}_{2}\right), 3.39$ (d, $\left.2 \mathrm{H}, \mathrm{NCH}_{2} \mathrm{CH}=\right), 4.20$ (t, $1 \mathrm{H}$, $4-\mathrm{H}), 5.11\left(\mathrm{dd},{ }^{3} \mathrm{~J}=10.3,{ }^{2} \mathrm{~J}=1.6 \mathrm{~Hz}, 1 \mathrm{H}, \mathrm{CH}_{2} \mathrm{CH}=\mathrm{CH}_{2}\right.$ ), 
$5.18\left(\mathrm{dd},{ }^{3} \mathrm{~J}=17.1,{ }^{2} \mathrm{~J}=1.8 \mathrm{~Hz}, 1 \mathrm{H}, \mathrm{CH}_{2} \mathrm{CH}=\mathrm{CH}_{2}\right)$, $5.83\left(\mathrm{mc}, 1 \mathrm{H}, \mathrm{NCH}_{2} \mathrm{CH}=\right), 6.06$ (s, $1 \mathrm{H}, \mathrm{CH}=\mathrm{C}-$ Thienyl), $6.69\left(\mathrm{dd},{ }^{3} J=3.4,{ }^{4} J=1.2 \mathrm{~Hz}, 1 \mathrm{H}, 3-\mathrm{H}_{\text {thienyl }}\right), 6.94(\mathrm{dd}$, $\left.{ }^{3} J=5.0,{ }^{3} J=3.4 \mathrm{~Hz}, 1 \mathrm{H}, 4-\mathrm{H}_{\text {thienyl }}\right), 7.21\left(\mathrm{dd},{ }^{3} J=5.0\right.$, $\left.{ }^{4} J=1.0 \mathrm{~Hz}, 1 \mathrm{H}, 5-\mathrm{H}_{\text {thienyl }}\right) .-{ }^{13} \mathrm{C}$ NMR $(125.77 \mathrm{MHz})$ : $\delta=29.0(\mathrm{C}-3), 29.5\left(\mathrm{CMe}_{3}\right), 36.8\left(\mathrm{CMe}_{3}\right), 52.3(\mathrm{C}-2), 55.4$ $\left(\mathrm{NCH}_{2} \mathrm{CH}=\right), 104.2(\mathrm{C}-4), 116.6\left(\mathrm{CH}_{2} \mathrm{CH}=\mathrm{CH}_{2}\right), 119.9$ $(\mathrm{CH}=\mathrm{C}-\mathrm{Thienyl}), 124.4\left(\mathrm{C}-5_{\text {thienyl }}\right), 126.0\left(\mathrm{C}-4_{\text {thienyl }}\right), 126.5$ (C-3 $\left.3_{\text {thienyl }}\right), 135.8\left(\mathrm{NCH}_{2} \mathrm{CH}=\right), 140.3\left(\mathrm{C}-2_{\text {thienyl }}\right), 146.6$ ( $\mathrm{CH}=C$-Thienyl), 147.7 (s, C-5). $-\mathrm{C}_{17} \mathrm{H}_{23} \mathrm{NS}$ (273.44).

1-Allyl-6-[(Z)-2-(4-chlorophenyl)-3,3-dimethylbut-1-en$y l]-1,2,3,4$-tetrahydropyridine (19a): The synthesis was performed as described for 18a, from a solution of ( $t$ $\mathrm{Bu})_{2} \mathrm{CuLi} \cdot \mathrm{LiBr}(4.2 \mathrm{mmol})$ in THF $(10 \mathrm{ml})$ and a solution of iminium salt 17a [17] $(0.759 \mathrm{~g}, 2.0 \mathrm{mmol})$ in THF $(10 \mathrm{ml})$. A yellow oil was obtained which could not be purified further and was used directly (see below). Yield of crude 19a: $0.555 \mathrm{~g}(88 \%)$. - IR (film): $v=3074$ (w), 2960, 1686, 1591, 1487, 1392, 1245 (all m) $\mathrm{cm}^{-1} .-{ }^{1} \mathrm{H}$ NMR (500.14 MHz): $\delta=1.08(\mathrm{~s}, 9 \mathrm{H}, t \mathrm{Bu}$ ), 1.48 (quin, $2 \mathrm{H}$, 3- $\left.\mathrm{H}_{2}\right), 1.73\left(\mathrm{mc}, 2 \mathrm{H}, 4-\mathrm{H}_{2}\right), 2.79\left(\mathrm{t}, 2 \mathrm{H}, 2-\mathrm{H}_{2}\right), 3.48$ (d, $\left.2 \mathrm{H}, \mathrm{NCH}_{2} \mathrm{CH}=\right), 4.08(\mathrm{~s}, 1 \mathrm{H}, 5-\mathrm{H}), 5.09\left(\mathrm{~d},{ }^{3} \mathrm{~J}=\right.$ $\left.10.3 \mathrm{~Hz}, 1 \mathrm{H}, \mathrm{CH}_{2} \mathrm{CH}=\mathrm{CH}_{2}\right), 5.14\left(\mathrm{~d},{ }^{3} J=17.4 \mathrm{~Hz}, 1 \mathrm{H}\right.$, $\left.\mathrm{CH}_{2} \mathrm{CH}=\mathrm{CH}_{2}\right), 5.76\left(\mathrm{mc}, 1 \mathrm{H}, \mathrm{CH}_{2} \mathrm{CH}=\mathrm{CH}_{2}\right), 6.00(\mathrm{~s}, 1 \mathrm{H}$, $\mathrm{CH}=\mathrm{C}-\mathrm{Aryl}$ ), 6.94/7.21 (AA'BB', $\left.4 \mathrm{H}, \mathrm{H}_{\text {aryl }}\right) .-{ }^{13} \mathrm{C} \mathrm{NMR}$ (125.77 MHz): $\delta=21.1$ (C-3), 22.7 (C-4), $29.7\left(\mathrm{CMe}_{3}\right)$, $36.3\left(\mathrm{CMe}_{3}\right),(\mathrm{C}-2), 54.8\left(\mathrm{NCH}_{2} \mathrm{CH}=\right), 104.0$ (C-5), 116.1 $\left(\mathrm{CH}_{2} \mathrm{CH}=\mathrm{CH}_{2}\right), 123.3(\mathrm{CH}=\mathrm{C}-\mathrm{Aryl}), 127.0(\mathrm{~d}), 131.1(\mathrm{~d})$, $131.6(\mathrm{C}-\mathrm{Cl}), 136.4\left(\mathrm{CH}_{2} \mathrm{CH}=\mathrm{CH}_{2}\right), 138.6(\mathrm{~s}), 141.6(\mathrm{C}-6)$, $151.2\left(\mathrm{CH}=C\right.$-Aryl). $-\mathrm{C}_{20} \mathrm{H}_{26} \mathrm{ClN}$ (315.88).

1-Allyl-6-[(Z)-3,3-dimethyl-2-(thiophen-2-yl)but-1-enyl]-1,2,3,4-tetrahydropyridine (19b): As described above for 18a, the compound was prepared from a solution of $(t-\mathrm{Bu})_{2} \mathrm{CuLi} \cdot \mathrm{LiBr}(4.2 \mathrm{mmol})$ in THF $(10 \mathrm{ml})$ and a solution of iminium salt 17b [17] $(0.759 \mathrm{~g}, 2.0 \mathrm{mmol})$ in THF $(10 \mathrm{ml})$. A yellow oil was obtained which could not be purified further and was used directly (vide infra). Yield of 19b: $0.523 \mathrm{~g}$ (91\%). - IR (film): $v=3070$ (w), 2954, 2833, 1632, 1462, 1434, 1360, 1225, 1171, 1129 (all m) $\mathrm{cm}^{-1} .-{ }^{1} \mathrm{H}$ NMR $(500.14 \mathrm{MHz}): \delta=1.14(\mathrm{~s}, 9 \mathrm{H}, t \mathrm{Bu})$, 1.55 (quin, $\left.2 \mathrm{H}, 3-\mathrm{H}_{2}\right), 1.79\left(\mathrm{mc}, 2 \mathrm{H}, 4-\mathrm{H}_{2}\right), 2.84$ (t, $2 \mathrm{H}$, 2- $\left.\mathrm{H}_{2}\right), 3.48\left(\mathrm{~d}, 2 \mathrm{H}, \mathrm{NCH}_{2} \mathrm{CH}=\right), 4.29$ (s, br, $\left.1 \mathrm{H}, 5-\mathrm{H}\right)$, $5.10\left(\mathrm{dd},{ }^{3} \mathrm{~J}=10.2,{ }^{2} \mathrm{~J}=1.8 \mathrm{~Hz}, 1 \mathrm{H}, \mathrm{CH}_{2} \mathrm{CH}=\mathrm{CH}_{2}\right), 5.16$ (dd, ${ }^{3} J=17.1,{ }^{2} J=1.7 \mathrm{~Hz}, 1 \mathrm{H}, \mathrm{CH}_{2} \mathrm{CH}=\mathrm{CH}_{2}$ ), 5.80 (mc, $1 \mathrm{H}, \mathrm{NCH}_{2} \mathrm{CH}=$ ), 6.09 (s, $1 \mathrm{H}, \mathrm{CH}=\mathrm{C}-$ Thienyl), 6.71 (dd, $\left.{ }^{3} J=3.5,{ }^{4} J=1.1 \mathrm{~Hz}, 1 \mathrm{H}, 3-\mathrm{H}_{\text {thienyl }}\right), 6.92\left(\mathrm{dd},{ }^{3} J=5.1\right.$, $\left.{ }^{3} J=3.5 \mathrm{~Hz}, 1 \mathrm{H}, 4-\mathrm{H}_{\text {thienyl }}\right), 7.18\left(\mathrm{dd},{ }^{3} J=5.1,{ }^{4} J=1.2 \mathrm{~Hz}\right.$, $\left.1 \mathrm{H}, 5-\mathrm{H}_{\text {thienyl }}\right) .-{ }^{13} \mathrm{C} \mathrm{NMR}(125.77 \mathrm{MHz}): \delta=21.1(\mathrm{C}-3)$, 22.9 (C-4), $29.7\left(\mathrm{CMe}_{3}\right), 36.6\left(\mathrm{CMe}_{3}\right), 47.5(\mathrm{C}-2), 55.3$ $\left(\mathrm{NCH}_{2} \mathrm{CH}=\right), 103.4(\mathrm{C}-6), 116.2\left(\mathrm{CH}_{2} \mathrm{CH}=\mathrm{CH}_{2}\right), 123.8$ $\left(\mathrm{C}-5_{\text {thienyl }}\right), 125.8\left(\mathrm{C}-4_{\text {thienyl }}\right), 126.5(\mathrm{CH}=\mathrm{C}-\mathrm{Thienyl}), 126.7$ $\left(\mathrm{C}-3_{\text {thienyl }}\right), 136.6\left(\mathrm{NCH}_{2} \mathrm{CH}=\right), 140.8$ (s, C-2 thienyl $), 142.1$ (s, C-6), $145.0\left(\mathrm{CH}=\mathrm{C}-\right.$ Thienyl). $-\mathrm{C}_{18} \mathrm{H}_{25} \mathrm{NS}$ (287.46).
Dimethyl 1-allyl-2-[(Z)-2-(4-chlorophenyl)-3,3-dimethylbut-1-enyl]-6,7-dihydro-1H-azepine-3,4-dicarboxylate (21a): Freshly prepared crude aminodiene 18a $(0.520 \mathrm{~g}$, $1.7 \mathrm{mmol})$ was dissolved in ether $(15 \mathrm{ml})$ and cooled at $-70{ }^{\circ} \mathrm{C}$. DMAD $(0.233 \mathrm{ml}, 1.9 \mathrm{mmol})$ was added dropwise and the mixture was allowed to come to r.t. over $12 \mathrm{~h}$. The solvent was replaced by toluene $(5 \mathrm{ml})$ and the solution was heated at $120{ }^{\circ} \mathrm{C}$ during $5 \mathrm{~h}$ in a thick-walled Schlenk tube. Evaporation of the solvent left an oil which was first submitted to flash chromatography (10 $\mathrm{g}$ of silica gel, $\mathrm{CH}_{2} \mathrm{Cl}_{2}$ ), then to column chromatography (Merck Lobar columns, ether/petroleum ether $1: 4 \rightarrow 1: 1)$. Yield of 21a: $0.412 \mathrm{~g}$ (47\% rel. to iminium salt 16a); dark-yellow oil. ${ }^{1} \mathrm{H}$ NMR $(500.14 \mathrm{MHz}, 253 \mathrm{~K}): \delta=1.16(\mathrm{~s}, 9 \mathrm{H}, t \mathrm{Bu})$, $1.83-1.92(\mathrm{~m}, 1 \mathrm{H}, 6-\mathrm{H}), 2.23-2.41(\mathrm{~m}, 2 \mathrm{H}, 6-\mathrm{H}, 7-\mathrm{H})$, $2.85-2.93(\mathrm{~m}, 1 \mathrm{H}, 7-\mathrm{H}), 3.06\left(\mathrm{dd},{ }^{2} J=15.1,{ }^{3} J=8.1 \mathrm{~Hz}\right.$, $\left.1 \mathrm{H}, \mathrm{NCH}_{2} \mathrm{CH}=\right), 3.68\left(\mathrm{~s}, 3 \mathrm{H}, \mathrm{OCH}_{3}\right), 3.72\left(\mathrm{~s}, 3 \mathrm{H}, \mathrm{OCH}_{3}\right)$, $4.34\left(\mathrm{~d},{ }^{2} \mathrm{~J}=14.8 \mathrm{~Hz}, 1 \mathrm{H}, \mathrm{NCH}_{2} \mathrm{CH}=\right), 5.10-5.19(\mathrm{~m}$, $2 \mathrm{H}, \mathrm{CH}_{2} \mathrm{CH}=\mathrm{CH}_{2}$ ), $5.61\left(\mathrm{mc}, 1 \mathrm{H}, \mathrm{CH}_{2} \mathrm{CH}=\mathrm{CH}_{2}\right), 6.52$ (t, $1 \mathrm{H}, 5-\mathrm{H}), 6.56$ (s, $1 \mathrm{H}, \mathrm{CH}=\mathrm{C}-\mathrm{Aryl}), 6.80-7.00$ (broad coalescing signal, $\left.2 \mathrm{H}_{\text {aryl }}\right), 7.24-7.35\left(\mathrm{~m}, 2 \mathrm{H}, \mathrm{H}_{\text {aryl }}\right)$. ${ }^{13} \mathrm{C}$ NMR $(125.77 \mathrm{MHz}, 253 \mathrm{~K}): \delta=28.9\left(\mathrm{CMe}_{3}\right), 29.5$ (C-6), $36.2\left(\mathrm{CMe}_{3}\right), 51.1\left(\mathrm{OCH}_{3}\right), 52.1\left(\mathrm{OCH}_{3}\right), 54.8$ (C-7), $56.9\left(\mathrm{NCH}_{2} \mathrm{CH}=\right), 99.9(\mathrm{C}-3), 117.8\left(\mathrm{CH}_{2} \mathrm{CH}=\mathrm{CH}_{2}\right)$, $123.9(\mathrm{CH}=\mathrm{C}-\mathrm{Aryl}), 127.2$ (broadened d, o/m- $\left.\mathrm{C}_{\text {aryl }}\right), 132.2$ (s, $\left.2 \mathrm{C}, \mathrm{Cl}_{\text {aryl }}, \mathrm{C}-4\right), 134.0\left(\mathrm{CH}_{2} \mathrm{CH}=\mathrm{CH}_{2}\right), 135.1(\mathrm{~d}$, C-5), 137.7 (s, C-Cl), 151.6 (s, C-2), 158.0 (s, =C-Aryl), 169.1 (C=O), $170.4(\mathrm{C}=\mathrm{O}) . \quad-\mathrm{C}_{25} \mathrm{H}_{30} \mathrm{ClNO}_{4}$ (443.97): calcd. C 67.63, 6.81, N 3.15; found C 68.19, H 6.69, N 3.17 .

Dimethyl 1-allyl-2-[(Z)-3,3-dimethyl-2-(thiophen-2-yl)but-1-enyl]-6,7-dihydro-1H-azepine-3,4-dicarboxylate

(21b): The preparation was carried out as described for 21a, from crude aminodiene $\mathbf{1 8 b}(0.494 \mathrm{~g}, 1.8 \mathrm{mmol})$ and DMAD $(0.246 \mathrm{ml}, 2.0 \mathrm{mmol})$ in ether $(15 \mathrm{ml})$. Yield of 21b: $0.434 \mathrm{~g}$ (52\% rel. to iminium salt 16b); yellow oil. IR (film): $v=2950$ (m), 1720 (s), 1536 (m), 1434 (m), 1252 (s), $1123(\mathrm{~m}) \mathrm{cm}^{-1} .-{ }^{1} \mathrm{H} \mathrm{NMR}(500.14 \mathrm{MHz}, 253 \mathrm{~K})$ : $\delta=1.21(\mathrm{~s}, 9 \mathrm{H}, t \mathrm{Bu}), 2.02-2.12(\mathrm{~m}, 1 \mathrm{H}, 6-\mathrm{H}), 2.24-2.35$ (m, $1 \mathrm{H}, 6-\mathrm{H}), 2.53$ (pseudo-t, $1 \mathrm{H}, 7-\mathrm{H}), 2.88-2.97$ (m, $1 \mathrm{H}, 7-\mathrm{H}), 3.18\left(\mathrm{dd},{ }^{2} J=15.0 \mathrm{~Hz},{ }^{3} \mathrm{~J}=8.1 \mathrm{~Hz}, 1 \mathrm{H}\right.$, $\left.\mathrm{NCH}_{2} \mathrm{CH}=\right), 3.68\left(\mathrm{~s}, 3 \mathrm{H}, \mathrm{OCH}_{3}\right), 3.73\left(\mathrm{~s}, 3 \mathrm{H}, \mathrm{OCH}_{3}\right), 4.23$ (broadened, $\left.{ }^{2} \mathrm{~J} \approx 15 \mathrm{~Hz}, 1 \mathrm{H}, \mathrm{NCH}_{2} \mathrm{CH}=\right), 5.09-5.18(\mathrm{~m}$, $\left.2 \mathrm{H}, \mathrm{CH}_{2} \mathrm{CH}=\mathrm{CH}_{2}\right), 5.63\left(\mathrm{mc}, 1 \mathrm{H}, \mathrm{CH}_{2} \mathrm{CH}=\mathrm{CH}_{2}\right), 6.54$ (t, $1 \mathrm{H}, J=5.4 \mathrm{~Hz}, 5-\mathrm{H}), 6.63(\mathrm{~s}, 1 \mathrm{H}, \mathrm{CH}=\mathrm{C}-\mathrm{Thienyl}$ ), $6.70\left(\mathrm{dd},{ }^{3} J=3.5,{ }^{4} J=1.2 \mathrm{~Hz}, 1 \mathrm{H}, 3-\mathrm{H}_{\text {thienyl }}\right), 6.96(\mathrm{dd}$, $\left.{ }^{3} J=5.1,{ }^{3} J=3.5 \mathrm{~Hz}, 1 \mathrm{H}, 4-\mathrm{H}_{\text {thienyl }}\right), 7.22\left(\mathrm{dd},{ }^{3} J=5.1\right.$, $\left.{ }^{4} J=1.2 \mathrm{~Hz}, 1 \mathrm{H}, 5-\mathrm{H}_{\text {thienyl }}\right) .-{ }^{13} \mathrm{C}$ NMR $(125.77 \mathrm{MHz}$, $253 \mathrm{~K}): \delta=28.9\left(\mathrm{CMe}_{3}\right), 30.1(\mathrm{C}-6), 36.4\left(\mathrm{CMe}_{3}\right), 51.1$ $\left(\mathrm{OCH}_{3}\right), 52.1\left(\mathrm{OCH}_{3}\right), 54.6(\mathrm{C}-7), 56.7 \quad\left(\mathrm{NCH}_{2} \mathrm{CH}=\right)$, 100.0 (s, C-3), $118.1\left(\mathrm{CH}_{2} \mathrm{CH}=\mathrm{CH}_{2}\right), 124.5\left(\mathrm{C}-5_{\text {thienyl }}\right)$, 126.0 (d, $\left.2 \mathrm{C}, \mathrm{C}-3_{\text {thienyl }}, \mathrm{C}-4_{\text {thienyl }}\right), 126.4(\mathrm{CH}=\mathrm{C}-$ thienyl $)$, 132.1 (s, C-4), $134.1 \quad\left(\mathrm{CH}_{2} \mathrm{CH}=\mathrm{CH}_{2}\right), 134.9$ (d, C-5), 
Table 1. Summary of crystallographic data and structure refinement for compounds $\mathbf{1 0},(1 E, 2 E, 4 E)-\mathbf{1 4}, \mathbf{2 4}$, and 25.

\begin{tabular}{|c|c|c|c|c|}
\hline & 10 & $\left(1\left(2^{\prime}\right) E, 2 E, 4 E\right)-\mathbf{1 4}$ & 24 & 25 \\
\hline Empirical formula & $\mathrm{C}_{19} \mathrm{H}_{21} \mathrm{NO}_{4} \mathrm{~S}$ & $\mathrm{C}_{20} \mathrm{H}_{25} \mathrm{NO}_{4} \mathrm{~S}$ & $\mathrm{C}_{26} \mathrm{H}_{32} \mathrm{ClNO}_{4}$ & $\mathrm{C}_{24} \mathrm{H}_{31} \mathrm{NO}_{4} \mathrm{~S} \times 0.5 \mathrm{C}_{3} \mathrm{H}_{6} \mathrm{O}^{\mathrm{a}}$ \\
\hline Formula weight & 359.43 & 375.47 & 457.98 & 458.60 \\
\hline Temperature (K) & 293(2) & 293(2) & 293(2) & 293(2) \\
\hline Crystal size [mm] & $0.75 \times 0.50 \times 0.35$ & $0.60 \times 0.35 \times 0.25$ & $0.38 \times 0.19 \times 0.12$ & $0.54 \times 0.27 \times 0.11$ \\
\hline Crystal system & orthorhombic & triclinic & monoclinic & monoclinic \\
\hline Space group & $P b c a$ & $P-1$ & $P 2_{1} / n$ & $P 2_{1} / n$ \\
\hline$a[\AA]$ & $14.536(3)$ & $8.807(2)$ & $12.637(2)$ & $6.3962(4)$ \\
\hline$b[\AA]$ & $11.447(2)$ & $10.463(2)$ & $13.786(1)$ & $22.652(1)$ \\
\hline$c[\AA]$ & $22.273(4)$ & $10.776(2)$ & $14.149(2)$ & $17.276(1)$ \\
\hline$\alpha\left[^{\circ}\right]$ & 90 & $97.20(3)$ & 90 & 90 \\
\hline$\beta\left[^{\circ}\right]$ & 90 & $93.77(3)$ & $100.41(2)$ & $99.93(9)$ \\
\hline$\gamma\left[{ }^{\circ}\right]$ & 90 & $96.40(3)$ & 90 & 90 \\
\hline Volume $\left[\AA^{3}\right]$ & $3706.1(12)$ & $975.8(3)$ & $2424.4(5)$ & $2465.3(3)$ \\
\hline$Z$ & 8 & 2 & 4 & 4 \\
\hline$\rho_{\text {ber }}\left[\mathrm{g} \cdot \mathrm{cm}^{-3}\right]$ & 1.288 & 1.278 & 1.255 & 1.235 \\
\hline$\mu\left(\mathrm{Mo}-\mathrm{K}_{\alpha}\right)\left[\mathrm{cm}^{-1}\right]$ & 0.20 & 0.19 & 0.19 & 0.16 \\
\hline$\theta$ Range $\left[{ }^{\circ}\right]$ & $2.20-23.50$ & $1.91-24.00$ & $1.99-25.96$ & $2.16-25.92$ \\
\hline \multirow[t]{3}{*}{ Index ranges } & $-1 \leq h \leq 16$ & $-1 \leq h \leq 10$ & $-14 \leq h \leq 15$ & $-7 \leq h \leq 7$ \\
\hline & $-1 \leq k \leq 12$ & $-11 \leq k \leq 11$ & $-16 \leq k \leq 16$ & $-27 \leq k \leq 27$ \\
\hline & $-24 \leq l \leq 1$ & $-12 \leq l \leq 12$ & $-17 \leq l \leq 17$ & $-21 \leq l \leq 21$ \\
\hline Reflections collected & 3496 & 3730 & 18673 & 21317 \\
\hline Independent reflections $\left(R_{\text {int }}\right)$ & $27408(0.0302)$ & $3054(0.0345)$ & $4691(0.0707)$ & $4582(0.0277)$ \\
\hline Completeness to $\theta_{\max }[\%]$ & 99.9 & 99.8 & 98.8 & 95.6 \\
\hline Data / restraints / parameters & $2740 / 0 / 265^{b}$ & $3054 / 0 / 332^{c}$ & $4691 / 0 / 294^{\mathrm{d}}$ & $4582 / 3 / 303^{d}$ \\
\hline Goodness-of-fit on $F^{2}$ & 1.057 & 1.050 & 0.764 & 0.913 \\
\hline Final $R$ indices $[I>2 \sigma(I)]: R_{1}, w R_{2}{ }^{\mathrm{e}}$ & $0.0757,0.1869$ & $0.0615,0.1671$ & $0.0376,0.0714$ & $0.0395,0.1121$ \\
\hline$R$ Indices (all data): $R_{1}, w R_{2}{ }^{\mathrm{f}}$ & $0.1278,0.2224$ & $0.0842,0.1856$ & $0.0977,0.0816$ & $0.0568,0.1226$ \\
\hline Largest diff. peak and hole $\left[\mathrm{e} \cdot \AA^{-3}\right]$ & $0.49,-0.70$ & $0.48,-0.43$ & $0.14,-0.17$ & $0.33,-0.20$ \\
\hline
\end{tabular}

a Acetone hemisolvate; the acetone molecule is disordered around an inversion centre; b hydrogen atom positions at the azonine ring were taken from a $\Delta F$ map and refined freely; all other hydrogen positions were calculated and treated as riding on their bond neighbors; c all hydrogen atom positions were taken from a $\Delta F$ map and refined with isotropic temperature factors, except for $\mathrm{H}(18)(\mathrm{calculated}$ position, riding model); ${ }^{\mathrm{d}}$ hydrogen atoms were calculated geometrically and treated as riding on their bond neighbors; the $\mathrm{H}$ atoms of the disordered acetone molecule in $\mathbf{2 6}$ were not located; ${ }^{\mathrm{e}}$ refinement based on $F^{2}$ values; ${ }^{\mathrm{f}} R_{1}=\Sigma|| F_{\mathrm{o}}|-| F_{\mathrm{c}} \| / \Sigma\left|F_{\mathrm{O}}\right| ; w R_{2}=\left[\Sigma\left(w\left(F_{\mathrm{O}}{ }^{2}-\right.\right.\right.$ \left.\left.\left.${F_{\mathrm{c}}}^{2}\right)^{2}\right) / \Sigma w\left({F_{\mathrm{o}}}^{2}\right)^{2}\right]^{1 / 2}$.

139.0 (s, C-2 thienyl $), 146.5$ (s, =C-thienyl), 158.3 (s, C-2), $169.1(\mathrm{C}=\mathrm{O}), \quad 170.6 \quad(\mathrm{C}=\mathrm{O}) . \quad-\mathrm{C}_{23} \mathrm{H}_{29} \mathrm{NO}_{4} \mathrm{~S} \quad(415.55)$ : calcd. C 66.48, H 7.03, N 3.37; found C 66.68, H 6.95, N 3.58 .

Dimethyl 2-(tert-butyl)-2-(4-chlorophenyl)-2,3,7,8,9,10hexahydroazepino[1,2-a]azocine-11,12-dicarboxylate (24): The preparation was carried out as described for 21a, from crude aminodiene 19a $(0.555 \mathrm{~g}, 1.76 \mathrm{mmol})$ and DMAD $(0.233 \mathrm{ml}, 1.90 \mathrm{mmol})$ in ether $(15 \mathrm{ml})$. Conditions for thermolysis: toluene, $160{ }^{\circ} \mathrm{C}, 5 \mathrm{~h}$. The product was purified by flash chromatography ( $10 \mathrm{~g}$ of silica gel, $\mathrm{CH}_{2} \mathrm{Cl}_{2}$ ) and recrystallized from ethanol. Yield of 24: $0.626 \mathrm{~g}$ (68\% rel. to iminium salt 17a); light-yellow crystals, m.p. $124{ }^{\circ} \mathrm{C}$. IR (KBr): $v=3045(\mathrm{w}), 2952(\mathrm{~m}), 1731$ (s), 1713 (s), $1432(\mathrm{~m}), 1263(\mathrm{~s}) \mathrm{cm}^{-1} .-{ }^{1} \mathrm{H}$ NMR $(500.14 \mathrm{MHz}, 250 \mathrm{~K})$ : $\delta=0.85$ (broadened s, $9 \mathrm{H}, t \mathrm{Bu}$ ), $1.26-1.37(\mathrm{~m}, 2 \mathrm{H}, 8-\mathrm{H}$, 9-H), $1.63-1.76$ ( $\mathrm{m}, 1 \mathrm{H}, 8-\mathrm{H}), 1.86$ (quin, $1 \mathrm{H}, 9-\mathrm{H}), 2.40-$ $2.51(\mathrm{~m}, 2 \mathrm{H}, 3-\mathrm{H}, 10-\mathrm{H}), 2.58-2.70(\mathrm{~m}, 2 \mathrm{H}, 7-\mathrm{H}, 10-\mathrm{H})$, $2.92(\mathrm{~d}, J=15.2,8.4 \mathrm{~Hz}, 1 \mathrm{H}, 3-\mathrm{H}), 3.27$ (virtual t, $1 \mathrm{H}, 7-$ $\mathrm{H}), 3.82\left(\mathrm{~s}, 3 \mathrm{H}, \mathrm{OCH}_{3}\right), 3.94\left(\mathrm{~s}, 3 \mathrm{H}, \mathrm{OCH}_{3}\right), 4.53$ (virtual t, $1 \mathrm{H}, 4-\mathrm{H}), 5.50\left(\mathrm{~d},{ }^{3} \mathrm{~J}=9.8 \mathrm{~Hz}, 1 \mathrm{H}, 5-\mathrm{H}\right), 5.54(\mathrm{~s}, 1 \mathrm{H}$, $1-\mathrm{H}), 7.12-7.24\left(\mathrm{~m}, 4 \mathrm{H}, \mathrm{H}_{\text {aryl }}\right) .-{ }^{13} \mathrm{C}$ NMR $(125.77 \mathrm{MHz}$, $250 \mathrm{~K}$ ): $\delta=23.2$ (t, C-8), 24.9 (t, C-9), 25.8 (broadened q, $\left.\mathrm{CMe}_{3}\right) 25.8(\mathrm{t}, \mathrm{C}-10), 31.7(\mathrm{t}, \mathrm{C}-3), 36.7\left(\mathrm{CMe}_{3}\right), 50.0(\mathrm{t}$, $\mathrm{C}-7), 52.6\left(\mathrm{OCH}_{3}\right), 52.7\left(\mathrm{OCH}_{3}\right), 54.7(\mathrm{~s}, \mathrm{C}-2), 102.3(\mathrm{~d}, \mathrm{C}-$ 4), 119.3 (d, C-1), 126.1/126.5 (both d, o-C aryl), 129.7 (d, C-5), 130.4 (s, C-Cl), 130.8/132.8 (both d, m-C $\mathrm{m}_{\text {aryl }}$ ), 134.9 (s, C-11), 137.0 (s, C-12a), 140.3 (s, C-12), 142.7 (s, C$\left.1_{\text {aryl }}\right), 167.7(\mathrm{C}=\mathrm{O}), 170.0(\mathrm{C}=\mathrm{O}) .-\mathrm{C}_{26} \mathrm{H}_{32} \mathrm{ClNO}_{4}(458.00)$ : calcd. C 68.19, H 7.04, N 3.06; found C 68.00, H 7.17, N 2.97.

13-(tert-Butyl)-3a,4,4a,5,7,8,9,12b-octahydroazocino[2,1-a]thieno[3,2-f]isoindole-11,12-dicarboxylate (25): The preparation was carried out as described for 21a, from crude aminodiene 19b $(0.523 \mathrm{~g}, 1.8 \mathrm{mmol})$ and DMAD $(0.246 \mathrm{ml}$, $2.0 \mathrm{mmol})$ in ether $(15 \mathrm{ml})$. Conditions for thermolysis: toluene, $160{ }^{\circ} \mathrm{C}, 5 \mathrm{~h}$. The product was purified by flash chromatography (10 $\mathrm{g}$ of silica gel, $\mathrm{CH}_{2} \mathrm{Cl}_{2}$ ) followed by column chromatography (silica gel, ethyl acetate/petroleum ether 1:3) and was recrystallized from acetone/petroleum 
ether. Yield of 25: $0.364 \mathrm{~g}(42 \%$ rel. to iminium salt 17b); light-yellow crystals, m.p. $124{ }^{\circ} \mathrm{C}$. - IR (KBr): $v=2940(\mathrm{~m}), 1713$ (s), 1550 (s), 1241 (vs) $\mathrm{cm}^{-1}$. ${ }^{1} \mathrm{H}$ NMR (500.14 MHz): $\delta=1.08-1.19(\mathrm{~m}, 1 \mathrm{H}, 8-\mathrm{H})$, $1.33(\mathrm{~s}, 9 \mathrm{H}, t \mathrm{Bu}), 1.35-1.45(\mathrm{~m}, 1 \mathrm{H}, 4-\mathrm{H}), 1.67-1.83(\mathrm{~m}$, $2 \mathrm{H}, 8-\mathrm{H}, 4-\mathrm{H}), 1.92-2.01(\mathrm{~m}, 1 \mathrm{H}, 4 \mathrm{a}-\mathrm{H}), 2.29-2.47$ (m, $2 \mathrm{H}, 9-\mathrm{H}), 2.87$ (dd, $J=14.1,3.6 \mathrm{~Hz}, 1 \mathrm{H}, 7-\mathrm{H}), 3.04$ (d, $J=9.8 \mathrm{~Hz}, 1 \mathrm{H}, 5-\mathrm{H}), 3.57$ (dd, $J=9.8,4.9 \mathrm{~Hz}, 1 \mathrm{H}, 5-\mathrm{H})$, $3.52\left(\mathrm{~s}, 3 \mathrm{H}, \mathrm{OCH}_{3}\right), 3.69\left(\mathrm{~s}, 3 \mathrm{H}, \mathrm{OCH}_{3}\right), 3.98(\mathrm{t}, 1 \mathrm{H}, 7-\mathrm{H})$, $4.17-4.24(\mathrm{~m}, 1 \mathrm{H}, 3 \mathrm{a}-\mathrm{H}), 4.30(\mathrm{~d}, J=6.0 \mathrm{~Hz}, 1 \mathrm{H}, 12 \mathrm{~b}-\mathrm{H})$, $5.63(\mathrm{dd}, J=6.2,2.8 \mathrm{~Hz}, 1 \mathrm{H}, 3-\mathrm{H}), 6.14-6.20(\mathrm{~m}, 2 \mathrm{H}$, 2-H, 10-H). $-{ }^{13} \mathrm{C}$ NMR (125.77 MHz): $\delta=13.5$ (t, C-8), 24.9 (t, C-9), $28.7\left(\mathrm{CMe}_{3}\right), 31.3$ (d, C-4a), $36.0\left(\mathrm{CMe}_{3}\right)$, 37.6 (t, C-4), 44.5 (t, C-7), 50.9 (d, C-3a), $51.1\left(\mathrm{OCH}_{3}\right), 51.5$ (d, C-12b), $51.9\left(\mathrm{OCH}_{3}\right), 59.6$ (t, C-5), 89.4 (s, C-12), 124.0 (d, C-2), 125.6 (d, C-3), 131.3 (d, C-10), 135.2 (s, 2 C, C-11, C-13a), 135.4 (s, C-13), 159.9 (s, C-12a), $169.6(\mathrm{C}=\mathrm{O})$, $169.9(\mathrm{C}=\mathrm{O}) .-\mathrm{C}_{24} \mathrm{H}_{31} \mathrm{NO}_{4} \mathrm{~S}$ (429.57): calcd. C 67.10, H 7.27, N 3.26; found C 66.03, H 7.29, N 3.02.

\section{$X$-ray crystal structure determinations}

Data collection was performed on a four-circle diffractometer (Siemens P4) for $\mathbf{1 0}$ and $\left(1\left(2^{\prime}\right) E, 2 E, 4 E\right)-\mathbf{1 4}$ and on an imaging-plate diffractometer (STOE IPDS) for $\mathbf{2 4}$ and 25, using monochromatized Mo- $K_{\alpha}$ radiation. No absorption correction was applied. The structures were solved by direct methods and refined by a full-matrix least-squares procedure based on $F^{2}$ values using the program package SHELX-97 [20]. Crystallographic data and refinement details are given in Table 1.

CCDC-281834 (10), $-281835 \quad\left(1\left(2^{\prime}\right) E, 2 E, 4 E-14\right)$, -281836 (24) and -281837 (25) contain the supplementary crystallographic data for this paper. These data can be obtained free of charge at www.ccdc.cam.ac.uk/conts/ retrieving.html [or from the Cambridge Crystallographic Data Centre, 12, Union Road, Cambridge CB2 1EZ, UK; fax: (internat.) +44-1223/336-033; E-mail: deposit@ ccdc.cam.ac.uk].
[1] (a) D. N. Reinhoudt, W. Verboom, G. W. Visser, W.P. Trompenaars, S. Harkema, G. J. van Hummel, J. Am. Chem. Soc. 106, 1341 (1984); (b) G. J.M. Vos, P.H. Benders, D. N. Reinhoudt, R. J.M. Egberink, S. Harkema, G. J. van Hummel, J. Org. Chem. 51, 2004 (1986).

[2] P. A. Evans, A. B. Holmes, Tetrahedron 47, 9131 (1991).

[3] Azepines: (a) R.M. Acheson, J.N. Bridson, T.S. Cameron, J. Chem. Soc., Perkin Trans. 1968 (1972); (b) K. Matsumoto, S. Nakamura, R. M. Acheson, Heterocycles 14, 1959 (1980); (c) H. Wamhoff, G. Hendrikx, Chem. Ber. 118, 863 (1985); (d) H. Wamhoff, Adv. Heterocycl. Chem. 38, 299 (1985).

[4] Azonines: (a) M. Acheson, G. Paglietti, P. A. Tasker, J. Chem. Soc., Perkin Trans. 12496 (1974); (b) B. Weinstein, L.-C. C. Lin, F. W. Fowler, J. Org. Chem. 45, 1657 (1980); (c) P. Sanna, A. Carta, G. Paglietti, J. Chem. Res. Synop. 16 (1992); (d) S. J. Taylor, A. M. Taylor, S. L. Schreiber, Angew. Chem. 116, 1713 (2004); (e) A.C. L. Badaró Trindade, D. C. dos Santos, L. Gil, C. Marazano, R. P. de Freitas Gil, Eur. J. Org. Chem. 1052 (2005).

[5] 7-, 8-, and 9-membered azaheterocycles: G. Maas, H.-G. Herz, E. Scheppach, B. S. Palm, H.-J. Schneider, Z. Naturforsch, 59b, 486 (2004).

[6] G. Maas, R. Reinhard, R. Neumann, M. Glaser, J. Prakt. Chem. 338, 441 (1996).

[7] These results were taken from the Doctoral Thesis of R. Reinhard (University of Kaiserslautern, 1994) and H. G. Herz (University of Ulm, 1999).
[8] K.C. Brannock, R.D. Burpitt, V.W. Goodlet, J.G. Thweatt, J. Org. Chem. 29, 818 (1964).

[9] W. Verboom, G. W. Visser, W. P. Trompenaars, D. N. Reinhoudt, S. Harkema, G. J. van Hummel, Tetrahedron 37, 3525 (1981).

[10] M. Brunner, R. Reinhard, R. Rahm, G. Maas, Synlett 627 (1994).

[11] (a) D. N. Epiotis, Angew. Chem. 86, 825 (1974); Angew. Chem., Int. Ed. Engl. 13, 751 (1974); (b) B. K. Carpenter, Tetrahedron 34, 1877 (1978).

[12] R. Criegee, D. Seebach, R. E. Winter, B. Börretzen, H.A. Brune, Chem. Ber. 98, 2339 (1965).

[13] (a) N. J. Leonard, R.C. Fox, M. Oki, J. Am. Chem. Soc. 76, 5708 (1954); (b) G. Spanka, P. Rademacher, J. Org. Chem. 51, 592 (1986).

[14] R. Griffith, J. B. Bremner, S. J. Titmuss, J. Comput. Chem. 18, 1211 (1997).

[15] E. D. Edstrom, J. Am. Chem. Soc. 113, 6690 (1991).

[16] (a) A. Sudau, W. Münch, J. W. Bats, U. Nubbemeyer, Chemistry 7, 611 (2001); (b) A. Sudau, W. Münch, J. W. Bats, U. Nubbemeyer, Eur. J. Org. Chem. 3304 (2002).

[17] R. Neumann, H.-G. Herz, G. Maas, J. Prakt. Chem. 341, 121 (1999).

[18] G. Zecchi, Synthesis 181 (1991).

[19] C. J. Roxburgh, Tetrahedron 50, 13199 (1994).

[20] G. M. Sheldrick, SHELX-97 - Program for the solution and refinement of crystal structures from diffraction data, University of Göttingen, Germany (1997). 